\title{
ON THE FACTORIZATION OF EXPRESSIONS OF VARIOUS TYPES*
}

\author{
BY \\ HENRY BLUMBERG
}

\section{INTRODUCTION}

Not a few mathematicians have dealt with the problem of setting up criteria by means of which the irreducibility of certain expressions in certain domains may be seen at a glance from the character of the expressions. Gauss, Kronecker, Schoenemann, Eisenstein, Dedekind, Floquet, Koenigsberger, Netto, Perron, M. Bauer, and Dumas have written on the subject. $\dagger$ A simple example of such criteria is the well-known Schoenemann-Eisenstein theorem, which is essentially as follows: $\ddagger$ If the polynomial

$$
C=y^{n}+c_{1} y^{n-1}+\cdots+c_{n},
$$

with integral coefficients, is such that a fixed prime $p$ goes into every

$$
c_{\nu}\{\nu=1,2, \cdots, n\},
$$

but $p^{2}$ does not go into $c_{n}$, then $C$ is irreducible in the domain of rational numbers. This theorem, while comparatively not rich in content, may be regarded as the nucleus of the work of the authors mentioned.

With the exception of Floquet and Koenigsberger, the authors mentioned deal exclusively with the case where the expressions are polynomials and chiefly with the case of polynomials whose coefficients belong to the domain

* Presented to the Society in less general form, November 28 and December 29, 1914.

† Gauss, Disquisitiones arithmeticae (1801), Art. 341; Kronecker, J o u r n a l f ü r M a t h e matik, vol. 29 (1845), p. 280, vol. 100 (1887), p. 79, Journal de Mathémati qu es, vol. 19 (1854), p. 177, ser. 2, vol. 1 (1856), p. 399; Schoenemann, J o u rnal für Math e matik, vol. 32 (1846), p. 100, vol. 40 (1850), p. 188; Eisenstein, J o u r a 1 für Mathematik, vol. 39 (1850), p. 160; Dedekind, Journal für Mathematik, vol. 54 (1857), p. 27; Floquet, Annales de l'école normale (1879) [cited by Koenigsberger]; Koenigsberger, Journal für Mathematik, vol. 115 (1895), p. 53, vol. 121 (1900), p. 320, Mathematische Annalen, vol. 53 (1900), p. 49; Netto, M a the matis che Annalen, vol. 48 (1897), p. 81 and Algebra (1896), vol. 1, lecture 5; Perron, M a th e mat is c he Annalen, vol. 60 (1905), p. 448, J o u r nalf ür Mathematik, vol. 132 (1907), p. 288; M. Bauer, J o urnalfür Mathe m a tik, vol. 128 (1905), p. 87 and p. 298, vol. 132 (1907), p. 21, vol. 134 (1908), p. 15; Dumas, J o urnal de Mathématiques, ser. 6, vol. 2 (1906), p. 191.

$\ddagger$ Schoenemann and Eisenstein, 1. c. This theorem has been wrongly attributed to Eisenstein alone by most of the mathematicians who have had occasion to refer to it. 
of rational numbers. Floquet and Koenigsberger have extended the investigation to linear homogeneous differential expressions.

A variety of methods have been employed. Thus the theory of algebraic fields, the theory of algebraic functions and the character of the solutions of linear homogeneous differential equations have been used.* Elementary methods, requiring no such means and based on the study of the expression as such without regard to the character of the solutions of the equation obtained by setting the expression equal to zero, have mostly succeeded in yielding only the less general results. $\dagger$

The principal aim of the present paper is to communicate a general theorem and certain immediate consequences of it, which include as special cases, with minor exceptions, $\ddagger$ all the results heretofore obtained in the indicated field of investigation. This theorem is given in $\S 6$, and for convenience of designation referred to as the "Theorem on the Product"; its direct consequences are the "Theorem on Common Divisors" (§6), the "Factorization Theorem" and the "Criterion for Irreducibility" ( $(7)$. Thus the work on the subject before the publication of the Schoenemann-Eisenstein theorem is summarized and generalized by that theorem, which is an exceedingly special case of the "Criterion for Irreducibility." Moreover, the Floquet theorem, all the results in the first and the third papers of Koenigsberger, the chief results in his second paper, all the results of Netto, all the results in the 1905 paper of Perron, the chief results in his 1907 paper and the chief results of Bauer and of Dumas come under this "Theorem on the Product."§

A former note\| was intended to give a summary of the present paper. Since then, however, the author has found how to unify and to generalize his previous results by means of the present theory. The note materially extends most of the known results in the field under discussion. For further details, the reader is referred to the note itself. Its relation to the present paper may be approximately described as follows: While the "Theorem on the Product" asserts that every pair $\left(m_{\zeta}, t_{\zeta}\right),\{\zeta=1,2, \cdots, h\}$, of the characteristic set $S_{C}$ satisfies the condition imposed upon it by the relation $S_{A}+S_{B}=S_{C}$, the note makes use merely of the fact that the first pair satisfies this condition. I Attention is also directed to the replacing of the three theories of the note by the single theory $\mathfrak{T}_{e}$ of the present paper.

* Heretofore the results have not been carried over into the field of difference equations, possibly because of the recency of the publication of existence theorems in that field.

† Dumas's memoir, l. c., is a notable exception.

$\ddagger$ For example, theorem I of Perron's 1907 paper, 1. c., p. 291.

\$ See previous citations.

IIProceedings of the National Academy of Sciences, June, 1915, pp. 374-381.

If See sections 4, 5, and 6. 
The large variety of cases covered necessitates an abstract, postulational treatment. The different special theories (for polynomials with various kinds of coefficients and for difference and differential expressions with various kinds of coefficients) are thus made to appear as instances of an abstract general theory $\mathfrak{T}_{e}$ (see $\S \S 3$ and 8). Only in such fashion can the interconnection of the more special results be brought into clear light and their unification effected. This abstract treatment is, moreover, especially fitting here, because a small number of simple assumptions is sufficient for the foundations of the theory.

The "Theorem on the Product" applies to Situations 4, 5 (for $k>1$ ), $6,7,8,9(\S 3)$, not treated before, as far as the author knows, and enriches in a marked degree the results for Situations $3(\S 3)$ and $10(\S 8)$ already studied. Nevertheless, it does not go beyond the results of Dumas for the case of polynomials whose coefficients are rational numbers or Hensel $p$-adic numbers. Our general theory may, in fact, be regarded as an extension to an abstract situation of Dumas's theorem, l. c., p. 217.*

The remarks at the close of $\S 7$ indicate the great difficulty in obtaining a more exhaustive criterion for irreducibility than the one actually obtained.

A sharp distinction has been made in the literature (as regards polynomials) between those investigations that are based on the divisibility properties of the coefficients and those that proceed from the consideration of the magnitude of the coefficients. $\dagger$ One of the interesting results is that the gap between these two types of investigation is bridged, both appearing subordinate to our general theory.

It is easy to see, as Koenigsberger has pointed out by an example, that the Schoenemann-Eisenstein theorem cannot be directly extended to the case of linear homogeneous differential expressions. Our abstract treatment furthermore lays bare the underlying reason-by no means evident otherwisewhy it breaks down, and at once indicates what analogous theorem may replace it. The "Criterion for Irreducibility" may, in fact, be properly regarded as a highly generalized Schoenemann-Eisenstein theorem for the

\footnotetext{
* It is interesting in this connection to compare several remarks in Perron's 1907 paper, 1. c. Referring to the work of Dumas on polynomials with rational coefficients as related to that of his predecessors, Perron says (p. 288): "Zu etwas allgemeineren Resultaten ist neuerdings Herr G. Dumas gelangt." Later on in the same paper (p. 304), Perron designates his most general result, Theorem VIII, as "ein sehr allgemeines Kriterium." The italics are in both cases my own. It turns out, however, that Perron's criterion is a special case of our theory, which is a natural and easy generalization of Dumas's results.

† Cf. Perron, 1907, l. c., p. 288 and Loewy in Pascal's' Repertorium (1910), Analysis II, pp. 292 and 293. The same kind of distinction may, of course, be made with regard to differential and other expressions.
} 
case of linear homogeneous differential expressions with coefficients taken as in Situation 6,7 , or 10 . $^{*}$

Here is a brief summary of the contents: Section 1 shows by means of an illustrative example how the Theory $\mathfrak{T}_{-1}$ of Section 2 arises. Section 3 describes various concrete situations falling under the Theory $\mathfrak{I}_{-1}$. Section 4 defines "characteristic set"-a fundamental notion for our purposes-and Section 5, the "sum of two characteristic sets." Section 6 gives the proof of the "Theorem on the Product" by the aid of two lemmas (established in $\$ \$ 4$ and 5). In Section 7, the "Factorization Theorem" and the "Criterion for Irreducibility" are obtained. Section 8 introduces the abstract theory $\mathfrak{T}_{\mathrm{\epsilon}}$ for every real $\epsilon$ and for $\epsilon=-\infty$. The appendix gives concrete examples of our results, at the same time exhibiting many instances of contact with the literature.

To secure greater clearness, nearly all the letters used are made to have a fixed meaning throughout the paper. Thus, for example, throughout the paper, $A, B, C$ stand respectively for $\left(a_{0}, a_{1}, \cdots, a_{r}\right),\left(b_{0}, b_{1}, \cdots, b_{s}\right)$, $\left(c_{0}, c_{1}, \cdots, c_{n}\right) ; \alpha_{\lambda}, \beta_{\mu}, \gamma_{\nu}$, respectively for the "rank" of $a_{\lambda}, b_{\mu}, c_{\nu}$ (see $\S 2) ;\left\{\left(m_{1}, t_{1}\right),\left(m_{2}, t_{2}\right), \cdots,\left(m_{h}, t_{h}\right)\right\}$, for the " characteristic set" $S_{C}$ of $C$ (see $\$ 4$ ); etc. Slight departures from this state of affairs will be evident from the context.

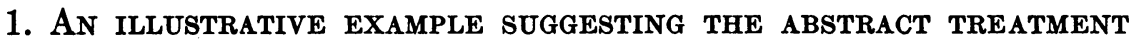

Instead of proceeding directly to the exposition of the abstract theory mentioned in the introduction, we shall foreshadow the abstract situation by drawing certain pertinent conclusions in regard to one of its concrete instances. $\dagger$ In this way, we believe, the assumptions used in $\S 2$ will be suffieiently motivated for the reader.

Let $\mathfrak{S}$ denote the class of rational functions of $x$ with complex numerical coefficients; and let

$$
C=c_{0}(x) \frac{d^{n} y}{d x^{n}}+c_{1}(x) \frac{d^{n-1} y}{d x^{n-1}}+\cdots+c_{n}(x) y
$$

be a linear homogeneous differential expression whose coefficients belong to $\mathbb{S}$. We are concerned with the representation of $C$ as a product $A \cdot B$, where

$$
A=a_{0}(x) \frac{d^{r} y}{d x^{r}}+a_{1}(x) \frac{d^{r-1} y}{d x^{r-1}}+\cdots+a_{r}(x) y
$$

* Curiously, Koenigsberger himself has obtained results (essentially for Situation 10, $\S 8$ ) that may be properly regarded as extensions of the Schoenemann-Eisenstein theorem to differential expressions. Lacking the abstract background, he failed, however, to notice this relation.

†The instance given has actually been the starting point of the author's present investigations. 
and

$$
B=b_{0}(x) \frac{d^{s} y}{d x^{8}}+b_{1}(x) \frac{d^{s-1} y}{d x^{8-1}}+\cdots+b_{s}(x) y,
$$

the coefficients $a_{\lambda}$ and $b_{\mu}$ belonging to $\mathfrak{S}$. As usual in such cases, $A \cdot B$ means here the symbolic product of the two differential expressions, so that

$$
\begin{aligned}
A \cdot B & =a_{0} \frac{d^{r} B}{d x^{r}}+a_{1} \frac{d^{r-1} B}{d x^{r-1}}+\cdots+a_{r} B \\
& =a_{0} b_{0} \frac{d^{r+s} y}{d x^{r+s}}+\left(r a_{0} \frac{d b_{0}}{d x}+a_{1} b_{0}+a_{0} b_{1}\right) \frac{d^{r+s-1} y}{d x^{r+s}-1}+\cdots \\
& =\sum_{\nu=0}^{r+8=n} c_{\nu} \frac{d^{n-\nu} y}{d x^{n-\nu}}
\end{aligned}
$$

where

$$
\begin{gathered}
c_{\nu}=\sum_{\mu=0}^{\nu} \sum_{\lambda=0}^{\nu-\mu}\left(\begin{array}{c}
r^{-}-\lambda \\
\nu-\lambda-\mu
\end{array}\right) a_{\lambda} \frac{d^{\nu-\mu-\lambda} b_{\mu}}{d x^{\nu-\mu-\lambda}}, \\
\left(\begin{array}{c}
r-\lambda \\
\nu-\lambda-\mu
\end{array}\right)=\frac{(r-\lambda) !}{(\nu-\lambda-\mu) !(r-\nu+\mu) !} .
\end{gathered}
$$

Understanding, as usual, by the degree of a rational fraction the degree of its numerator minus the degree of its denominator, we desire to call attention to several evident relations between the degrees of the $c$ 's on the one hand and the degrees of the $a$ 's and of the $b$ 's on the other. From the expression of $c_{\nu}$ in terms of $a_{\lambda}$ and $b_{\mu}$, we see that $c_{\nu}$ is a sum of products of the form $h_{\lambda \mu \sigma} a_{\lambda} d^{\sigma} b_{\mu} / d x^{\sigma}$, where $\lambda+\mu+\sigma=\nu$ and $h_{\lambda \mu \sigma}$ is a numerical coefficient. We now use the following facts regarding the degrees of products, sums, and derivatives of rational fractions: (1) The degree of a product is equal to the sum of the degrees of the factors. (2) The degree of a sum is at most equal to the highest degree $d$ attained by its terms, and exactly equal to $d$ if only one term has the degree $d$. (3) The degree of $d f(x) / d x, f(x)$ being a rational fraction, is at most one less than the degree of $f(x)$. To make these laws hold without exception, we agree to set the degree of 0 equal to $-\infty$ and to calculate with $-\infty$ in the usual manner. Thus $-\infty \pm 1=-\infty$, $-\infty+(-\infty)=-\infty$, etc. Let $\alpha_{\nu}, \beta_{\nu}, \gamma_{\nu}$ denote the degrees of $a_{\nu}$, $b_{\nu}, c_{\nu}$. It is then clear that $\gamma_{\nu}$ is at most equal to the largest value attained by the numbers of the set $\alpha_{\lambda}+\beta_{\mu}-\sigma$, when $\lambda, \mu$, and $\sigma$ vary so as to satisfy the relation $\lambda+\mu+\sigma=\nu$. This fact we express conveniently as follows:*

* The fact that $\sigma \leqq r-\lambda$ really imposes more stringent conditions upon $\gamma_{\nu}$ than we use. Because of our weaker conditions, we gain not only generality but also the important advantage of symmetry, by virtue of which later proofs are materially shortened. Condition (b) below is also weaker than the equivalent of the second part of (2) above (regarding the degree of a sum). 
(a) $\gamma_{\nu} \leqq \max _{\lambda+\mu+\sigma=\nu}\left(\alpha_{\lambda}+\beta_{\mu}-\sigma\right),\{\nu=0,1,2, \cdots, n\}$.

Furthermore, suppose that for the fixed values $\lambda=\bar{\lambda}, \mu=\bar{\mu}, \sigma=0$ (where $\bar{\lambda}+\bar{\mu}=\nu$ ), $\alpha_{\bar{\lambda}}+\beta_{\bar{\mu}}$ is greater than $\alpha_{\lambda}+\beta_{\mu}-\sigma$ for every $[\lambda, \mu, \sigma]$ 丰 $[\bar{\lambda}, \bar{\mu}, 0]$ satisfying the relation $\lambda+\mu+\sigma=\nu$; it follows that $\gamma_{\nu}=\alpha_{\bar{\lambda}}$ $+\beta_{\bar{\mu}}$. Hence, in addition to $(a)$, we have:

(b) $\gamma_{\nu}=\alpha_{\bar{\lambda}}+\beta_{\bar{\mu}}\{\bar{\lambda}+\mu=\nu\}$ if $\alpha_{\bar{\lambda}}+\beta_{\bar{\mu}}>\alpha_{\lambda}+\beta_{\mu}-\sigma\{\lambda+\mu+\sigma=\nu\}$ for every $[\lambda, \mu, \sigma] \neq=1 \bar{\lambda}, \bar{\mu}, 0],\{\nu=0,1,2, \cdots, n\}$.

It will appear in the sequel that the simple and evident properties $(a)$ and (b) of linear homogeneous differential expressions with rational coefficients essentially suffice for the demonstration of our factorization results concerning such expressions. But the properties $(a)$ and $(b)$ are shared by many other expressions (see the instances in $\S 3$ ). To dispense with the duplication of proofs and for other reasons given in the introduction, we proceed (in the next section) to the exposition of an abstract theory $\mathfrak{T}_{-1}$, of which the illustrative example described in this section is a special instance.* The domain $\mathfrak{S}$ of rational functions will be replaced by an unrestricted aggregate $\mathfrak{S}$; the differential expressions, by "parentheses" of $\mathfrak{S}$; the degree of a rational function, by the "rank" of an element of $\mathfrak{5}$; and the product of two differential expressions, by the " product" of two " parentheses," the abstract " product" being subjected to postulates corresponding to the properties $(a)$ and $(b)$.

\section{The ABSTRACT TheORY $\mathfrak{T}_{-1}$}

Let $\mathfrak{S}$ denote any given aggregate whatsoever. $\dagger$ We shall deal with finite, ordered subaggregates $E=\left(e_{0}, e_{1}, e_{2}, \cdots, e_{m}\right)$ of $\mathfrak{S}, e_{\nu}\{\nu=0,1,2, \cdots, m\}$ being an element of $\mathfrak{S}$. Such a finite, ordered subaggregate $E$ we shall call a "parenthesis of $\mathbf{S} . "$ We assume that the "product"

$$
A \cdot B=\left(a_{0}, a_{1}, a_{2}, \cdots, a_{r}\right) \cdot\left(b_{0}, b_{1}, b_{2}, \cdots, b_{s}\right)
$$

of any two parentheses $A$ and $B$ of $\mathfrak{S}$ is equal to a parenthesis

$$
C=\left(c_{0}, c_{1}, c_{2}, \cdots, c_{n}\right)
$$

of $\mathfrak{S}$ for which $n=r+s$. We assume furthermore that with every element $e$ of $\subseteq$ there is associated a single number $\eta$ called the "rank" of $e$, $\eta$ being a real number or $-\infty$ (never $+\infty)$, $\ddagger$ such that when $A \cdot B=C$

* The reason for the notation " $\mathfrak{T}_{-1}$ " lies, as indicated in the introduction, in the fact that this abstract theory is later ( 8) exhibited as a special case of a more general theory $\mathfrak{T}_{\epsilon}$, the latter reducing to the former if $\epsilon=-1$.

$\dagger$ In regard to the use of the unrestricted aggregate (= general range) and several other features of this paper, such as the postulational unification of analogous theories, compare E. H. Moore, Introduction to a Form of General Analysis (1910), preface.

$\ddagger$ In all the concrete situations 1-10 incl., with the exception of $4 b, 7 b$, and $9 b, \eta$ happens to be always an integer. The proof of the "Theorem on the Product" is, however, not rendered more difficult if $\eta$ is merely restricted to being real. 
the following properties hold for the "ranks" $\alpha_{\lambda}, \beta_{\mu}, \gamma_{\nu}$ of $a_{\lambda}, b_{\mu}, c_{\nu},\{\nu=0$, $1,2, \cdots, n\}:^{*}$

(a) $\gamma_{\nu} \leqq \max _{\lambda+\mu+\sigma=\nu}\left(\alpha_{\lambda}+\beta_{\mu}-\sigma\right),\{0 \leqq \lambda \leqq r, 0 \leqq \mu \leqq s, 0 \leqq \sigma\}$.

(b) $\gamma_{\nu}=\alpha_{\bar{\lambda}}+\beta_{\bar{\mu}}\{\bar{\lambda}+\bar{\mu}=\nu\}$ if $\alpha_{\bar{\lambda}}+\beta_{\bar{\mu}}>\alpha_{\lambda}+\beta_{\mu}-\sigma\{\lambda+\mu+\sigma=\nu\}$ for every $[\lambda, \mu, \sigma]$ 丰 $[\bar{\lambda}, \bar{\mu}, 0],\{0 \leqq \lambda \leqq r, 0 \leqq \mu \leqq s, 0 \leqq \sigma\}$.

The "order" of the parenthesis $E \equiv\left(e_{0}, e_{1}, \cdots, e_{m}\right)$ is defined as $n-k$, where $k$ is such that $\eta_{0}=-\infty, \eta_{1}=-\infty, \cdots, \eta_{k-1}=-\infty$, and $\eta_{k} \neq-\infty$. If every $\eta_{\nu}\{\nu=0,1, \cdots, m\}$ is $-\infty$, the order of $E$ is defined to be 0 .

"Reducibility," "irreducibility," "factor," etc., are defined for $\mathfrak{I}_{-1}$ (and later for $\mathfrak{T}_{e}$ ) in expected fashion. Thus $C$ is said to be "reducible" if two parentheses $A$ and $B$ of $\subseteq$ exist, such that $r \geqq 1, s \geqq 1$ and $C=A \cdot B$.

\section{INSTANCES OF THE ABSTRACT THEORY $\mathfrak{T}_{-1}$}

We shall now describe various important situations that fall under the abstract theory $\mathfrak{I}_{-1}$. For this purpose, we shall define in each case the aggregate $\mathfrak{S}$, the parentheses of $\mathfrak{S}$, the product of two parentheses and the rank of every element of $\subseteq$. The verification of the properties $(a)$ and $(b)$ of $\S 2$ for the instances given below is left to the reader.

Situation 1. $\subseteq$ consists of the set of rational numbers. We understand by the parenthesis $\left(e_{0}, e_{1}, e_{2}, \cdots, e_{m}\right)$ of $\subseteq$ the polynomial

$$
e_{0} y^{m}+e_{1} y^{m-1}+\cdots+e_{m}
$$

in the letter $y$. The product of two parentheses

$$
\left(a_{0}, a_{1}, \cdots, a_{r}\right) \cdot\left(b_{0}, b_{1}, \cdots, b_{s}\right)
$$

is defined, as usual, to be equal to the parenthesis $\left(c_{0}, c_{1}, \cdots, c_{n}\right)$, where $n=r+s$ and

$$
c_{0}=a_{0} b_{0}, \quad c_{1}=a_{0} b_{1}+a_{1} b_{0}, \quad \cdots, \quad c_{n}=a_{r} b_{s} .
$$

The rank of an element $e=e^{\prime} / e^{\prime \prime}$ (where $e^{\prime}$ and $e^{\prime \prime}$ are integers) is defined with reference to a fixed prime $p$. First let $e \neq 0$; let $e^{\prime}$ be divisible by $p^{d^{\prime}}$ but not by $p^{d^{\prime}+1} ; e^{\prime \prime}$, by $p^{d^{\prime \prime}}$ but not by $p^{d^{\prime \prime}+1}$. We then define the rank of $e$ by the equation $\eta=d^{\prime \prime}-d^{\prime}$. Moreover, we naturally enough define the rank of 0 to be $-\infty$.

Situation 2. $\subseteq$ consists of the class of Hensel's $p$-adic numbers. The parentheses of $\mathfrak{S}$ and the products of parentheses are defined as in 1. The

${ }^{*}$ Because of the nature of our assumptions for $\mathfrak{T}_{-1}$, which involve essentially only the ranks of the elements of $\mathfrak{S}$, it is possible to build up just as general a theory as $\mathfrak{T}_{-1}$ by dealing exclusively with parentheses whose elements are ranks and hence always real numbers or $-\infty$. We have here an example of an apparently very general theory (based on an unrestricted aggregate and involving few assumptions) that is equivalent to an apparently very special one (about parentheses of real numbers). 
rank of the $p$-adic number $e$ is the negative of Hensel's "order" of $e$ with respect to $p . *$

Situation 3. $\subseteq$ consists of the class of rational fractions

$$
e=e^{\prime}\left(x_{1}, \cdots, x_{k}\right) / e^{\prime \prime}\left(x_{1}, \cdots, x_{k}\right)
$$

in $k$ letters, $x_{1}, x_{2}, \cdots, x_{k}, e^{\prime}$ and $e^{\prime \prime}$ being polynomials with arbitrary complex numerical coefficients. $\dagger$ The parentheses and the products of parentheses are defined as in 1 . The rank of an element $e(\neq 0)$ is defined by the equation $\eta=d^{\prime}-d^{\prime \prime}$, where $d^{\prime}$ and $d^{\prime \prime}$ represent respectively the degrees (in the usual sense) of $e^{\prime}$ and $e^{\prime \prime}$ in the $k$ letters $x_{1}, x_{2}, \cdots, x_{k}$. The rank of 0 is defined to be $-\infty$.

Situation 4a. $\subseteq$ consists of the collectivity of elements $e=\sum_{\nu=0}^{\nu} v_{\nu} x^{\eta-\nu}$, where the $v$ 's are arbitrary complex numbers, $\eta$ is an integer and also $v_{0} \neq 0$ unless $e=0$; that is, $e$ is a Laurent series having only a finite number of terms with positive exponents. $\neq$ The parentheses and the products are defined as in 1. The rank of $e(\neq 0)$ is defined to be the exponent $\eta$ of $x$ in the first non-zero term of the development of $e$. The rank of 0 is $-\infty$.

Situation $4 b$. $\subseteq$ consists of the collectivity of series $\sum_{\substack{\nu=\infty \\ \nu=0}} v_{\nu} x^{(\theta / \delta)-(\nu / \delta)}$ where $\theta$ and $\delta(>1)$ are integers; the elements of $\mathfrak{S}$ are thus descending series in integral powers of $x^{1 / 8}$. Otherwise, as in $4 a$.

Situation 5. $\subseteq$ consists of the collectivity of fractions $e=e^{\prime} / e^{\prime \prime}$, where $e^{\prime}$ and $e^{\prime \prime}$ are power series in $k$ letters $x_{1}, x_{2}, \cdots, x_{k}$ with arbitrary complex numerical coefficients:

$$
\begin{aligned}
e^{\prime} & =\sum_{\lambda_{1}, \cdots, \lambda_{k}=0}^{\infty} v_{\lambda_{1}, \cdots, \lambda_{k}}^{\prime} x_{1}^{\lambda_{1}} \cdots x_{k}^{\lambda_{k}}, \\
e^{\prime \prime} & =\sum_{\lambda_{1}, \cdots, \lambda_{k}=0}^{\infty} v_{\lambda_{1}, \cdots, \lambda_{k}}^{\prime \prime} x_{1}^{\lambda_{1}} \cdots x_{k}^{\lambda_{k}} .
\end{aligned}
$$

The parentheses and products are defined as in 1 . The rank of $e(\neq 0)$ is defined as $d^{\prime \prime}-d^{\prime}$, where $d^{\prime}$ and $d^{\prime \prime}$ represent the lowest degrees (in the usual sense) in the $k$ letters $x_{1}, \cdots, x_{k}$ of a non-zero term of $e^{\prime}$ and $e^{\prime \prime}$ respectively. The rank of 0 is $-\infty$.

Situation $6 . \S \subseteq$ consists of all rational fractions $e(x)=e^{\prime}(x) / e^{\prime \prime}(x)$, $e^{\prime}(x)$ and $e^{\prime \prime}(x)$ being polynomials with arbitrary complex numerical coef-

* See Hensel, J o u r n a l f ür M a the matik, vol. 127 (1904), pp. 51-84, or Zahlentheorie (1913), chapters 3 and 6.

$\dagger$ More generally, the numerical coefficients may belong to any abstract system $(K,+, \times)$, where $K$ is a class such that if $a$ and $b$ are elements of $K$ both $a+b$ and $a \times b$ are elements of $K$. This remark applies just as well to other situations below.

$\ddagger$ The question of convergence does not enter here because the formal character of the series is sufficient for our purposes. More generally, we may have similar series in two or more letters, the requisite change in the definition of rank being evident.

$\S$ Situation 6 is the illustrative example of $\S 1$. 
ficients. The parenthesis $\left(e_{0}, e_{1}, \cdots, e_{m}\right)$ is the linear homogeneous differential expression

$$
e_{0}(x) \frac{d^{m} y}{d x^{m}}+e_{1}(x) \frac{d^{m-1} y}{d x^{m-1}}+\cdots+e_{m}(x) y .
$$

The rank of $e$ is the degree of $e$ (in the usual sense), and the product of two parentheses is the symbolic product of the two corresponding differential expressions.

Situation 7a. $\quad$ S and rank are defined as in $4 a$; parentheses and products, as in 6.

Situation $7 b$. $\subseteq$ and rank are defined as in $4 b$; parentheses and products, as in 6.

Situation 8. 5 and rank are defined as in 6. The parenthesis

$$
\left(e_{0}, e_{1}, \cdots, e_{m}\right)
$$

of $\mathfrak{S}$ is the linear homogeneous difference expression

$$
e_{0} y_{x+m}+e_{1} y_{x+m-1}+\cdots+e_{m} y_{x} \text {. }
$$

The product $A \cdot B$ of two parentheses is the ordinary symbolic product of the corresponding difference expressions:

$$
A \cdot B=\left(a_{0}, a_{1}, \cdots, a_{r}\right) \cdot\left(b_{0}, b_{1}, \cdots, b_{s}\right)=\left(c_{0}, c_{1}, \cdots, c_{n}\right),
$$

where $n=r+s$ and

$$
c_{\nu}=\sum_{\rho=0}^{\nu} a_{\rho}(x) b_{\nu-\rho}(x+r-\rho), \quad\{\nu=0,1,2, \cdots, n\} .
$$

Situation $9 a$. $\subseteq$ and rank are defined as in $4 a$; parentheses and products, as in 8.

Situation $9 b$. $\subseteq$ and rank are defined as in $4 b$; parentheses and products, as in 8.

\section{4.* The characteristic set $S_{C}$ Belonging to $C$}

From now on (till $\S 8$ ), our discussion is based on the assumptions for the abstract theory $\mathfrak{I}_{-1}$ of $\S 2$. In the present section, however (and also in $\S 5$ ), we make use merely of the fact that every element of $\subseteq$ has a rank (which is a real number or $-\infty)$. $\dagger$

Let $C=\left(c_{0}, c_{1}, \cdots, c_{n}\right)$ be a given parenthesis of $\mathfrak{S}, \gamma_{\nu}$ being the rank of $c_{\nu}\{\nu=0,1, \cdots, n\}$. We assume throughout in what follows that $\gamma_{0}$ is finite

* At this point, the reader may pass directly to $\delta>$ ior the definition of $\mathfrak{T}_{e} ;$ he will thereby be enabled (on second reading, after $\S \S 4-7$ incl.) to verify more easily the statements of $\S 8$.

$\dagger$ On the other hand, if we have no regard to $(a)$ and $(b)$ of $\$ 2$, the definition of "characteristic set" here given appears highly artificial. 
(i. e., $\neq-\infty$ ). Let, moreover, $n \geqq 1$ (a condition to be subsequently dropped). With every such parenthesis $C$ we shall associate in unique fashion an ordered set $S_{C}$ of number pairs:

$$
S_{C}=\left\{\left(m_{1}, t_{1}\right),\left(m_{2}, t_{2}\right), \cdots,\left(m_{h}, t_{h}\right)\right\} .
$$

This set, which we call the "characteristic set belonging to $C$," we now define.* For short, we designate $\sum_{\nu=\{}^{\nu=\{} t_{\nu}$ by $s_{\zeta}\{\zeta=1,2, \cdots, h\} ; s_{0}=0$. We set $m_{1}$ equal to $\max _{\nu>0}\left(\overline{\gamma_{\nu}-\gamma_{0}} / \nu\right)$ except when this maximum is $<-1$; in the latter case, we set $m_{1}=-1$. If $m_{1}>-1$, we set $t_{1}$ equal to the last $\nu(\nu=1,2, \cdots, n)$ for which $\left(\gamma_{\nu}-\gamma_{0}\right) / \nu=m_{1}$; and if $m_{1}=-1$, we set $t_{1}=n$. $\dagger$ In general, we define $m_{\zeta+1}$ and $s_{\zeta+1}$ (and hence $t_{\zeta+1}$ ) for $\zeta=0,1, \cdots, h-1$ as follows:

$$
\begin{aligned}
& m_{\zeta+1}=\max _{\nu>s_{\zeta}}\left(\frac{\gamma_{\nu}-\gamma_{s_{\zeta}}}{\nu-s_{\zeta}},-1\right) . \\
& \left\{\begin{array}{l}
s_{\zeta+1}=\text { last } \nu>s_{\zeta} \text { such that } \frac{\gamma_{\nu}-\gamma_{s_{\nu}}}{\nu-s_{\zeta}}=m_{\zeta+1} \text {, if } m_{\zeta+1}>-1 \text {; and } \\
s_{\zeta+1}=n, \text { if } m_{\zeta+1}=-1 .
\end{array}\right.
\end{aligned}
$$

The following relations are direct consequences of the definition of $S_{C}$ :

(a) $s_{h}=\sum_{\nu=1}^{h} t_{\nu}=n ; \quad t_{\nu} \geqq 1 \quad\{\nu=1,2, \cdots, h\}$.

(b) $m_{1}>m_{2}>\cdots>m_{h} \geqq-1$.

(c) $\frac{\gamma_{\nu}-\gamma_{s_{\zeta}}}{\nu-s_{\zeta}}\left\{\nu>s_{\zeta}\right\} \leqq m_{\zeta+1}>\frac{\gamma_{\nu}-\gamma_{s_{\zeta}}}{\nu-s_{\zeta}}\left\{\nu>s_{\zeta+1}\right\}, \quad\{\zeta=0,1,2, \cdots, h-1\}$.

(d) $\gamma_{s_{\zeta}}-\gamma_{s_{\zeta-1}}=m_{\zeta} t_{\zeta}$ if $m_{\zeta}>-1$; hence

$$
\gamma_{s_{\zeta}}-\gamma_{0}=\sum_{\nu=1}^{\zeta} m_{\nu} t_{\nu} \text { if } m_{\zeta}>-1 \text {. }
$$

As in the theory of well-ordered sets, we call the set

* This "characteristic set" is the natural generalization of the "polygon of Newton" or of the "Puiseux numbers" for our abstract situation $\mathfrak{T}_{-1}$. The "generalized polygon of Newton" (for $\mathfrak{T}_{-1}$ ) may be essentially obtained by starting at the origin of a rectangular system of axes, and drawing a broken line such that the slope of the $\zeta$ th segment is $m_{\zeta}$ and its projection on the $X$-axis, $t_{\zeta}$. This broken line is, according to $1 b$ below, concave downward. The author might have made the entire treatment (including the proof of the "Theorem on the Product ") depend on the properties of this broken line; he has chosen, however, the equivalent analytical procedure. Cf. Hensel and Landsberg, Theorie der Algebraischen Funktionen einer Variabeln (1902), vierte Vorles., pp. 39-47 and Bauer, J o u rn a l f ü r M a th e mat ik, vol. 132, pp. 21-32.

$\dagger$ In the theory $\mathfrak{I}_{-1}$, the number -1 plays an exceptional rôle; in the theory $\mathfrak{T}_{e}$ (see $\S 8$ ) the number $\epsilon$ plays the corresponding rôle. 


$$
S_{C_{\zeta}}=\left\{\left(m_{1}, t_{1}\right), \cdots,\left(m_{\zeta}, t_{\zeta}\right)\right\}
$$

for $\zeta=1,2, \cdots, h$ a " segment" of $S_{C}$. The set $S_{C_{\zeta}}$ is evidently the characteristic set of the parenthesis $\left(c_{0}, c_{1}, \cdots, c_{s_{\zeta}}\right)$ of $\mathfrak{S}$. It is convenient in the sequel to adjoin the null-segment $0=S_{C_{0}}$ of $S_{C}$ and regard it as the characteristic set of every parenthesis $\left(c_{0}\right)$ of order 0 . As indicated above, we thus extend the definition of characteristic set to the case $n=0$. Every characteristic set has one and the same null-segment 0 .

LEMMA $*$ I. For every $(\nu, \zeta),\{\nu=0,1,2, \cdots, n ; \zeta=0,1,2, \cdots, h\}$ such that $m_{\zeta}>-1$, we have $\gamma_{\nu}-\gamma_{s_{\zeta}} \leqq\left(\nu-s_{\zeta}\right) m_{\zeta}$ and also $\leqq\left(\nu-s_{\zeta}\right) m_{\zeta+1}$.

Proof. If $\nu=s_{\zeta}$, the lemma is trivial. If $\nu>s_{\zeta}$ (whence $m_{\zeta+1}$ exists), the lemma follows from $1 c$ and $1 b$. If $\nu<s_{\zeta}$ (whence $m_{\zeta}$ exists), let $\omega$ be the uniquely determined integer satisfying the relations $s_{\omega-1} \leqq \nu<s_{\omega}$. Then $1 \leqq \omega \leqq \zeta$. According to the part of the lemma already established,

Moreover,

$$
\gamma_{\nu}-\gamma_{s_{\omega-1}} \leqq\left(\nu-s_{\omega-1}\right) m_{\omega}
$$

$$
\gamma_{s_{\zeta}}-\gamma_{s_{\omega-1}}=\sum_{\nu=\omega}^{\zeta} m_{\nu} t_{\nu}
$$

Hence

$$
\begin{aligned}
\gamma_{\nu}-\gamma_{\delta_{\zeta}} & \leqq\left(\nu-s_{\omega}\right) m_{\omega}-\sum_{\nu=\omega+1}^{\zeta} m_{\nu} t_{\nu} \\
& \leqq\left(\nu-s_{\omega}-\sum_{\nu=\omega+1}^{\zeta} t_{\nu}\right) m_{\zeta} \\
& =\left(\nu-s_{\zeta}\right) m_{\zeta} .
\end{aligned}
$$

Remark. If $\nu>s_{\zeta}, \gamma_{\nu}-\gamma_{s_{\zeta}}<\left(\nu-s_{\zeta}\right) m_{\zeta}$ (and, in fact, $<\left(\nu-s_{\zeta}\right) m$ for every $\left.m>m_{\zeta+1}\right)$; and if $\nu<s_{\zeta}, \gamma_{\nu}-\gamma_{s_{\zeta}}<\left(\nu-s_{\zeta}\right) m_{\zeta+1}$ (and, in fact, $<\left(\nu-s_{\zeta}\right) m$ for every $\left.m<m_{\zeta}\right)$.

\section{The SUM of Two Characteristic SEts}

Let $A=\left(a_{0}, a_{1}, \cdots, a_{r}\right)$ and $B=\left(b_{0}, b_{1}, \cdots, b_{s}\right)$ be two parentheses of $\dot{\mathfrak{S}}, \alpha_{\lambda}$ and $\beta_{\mu}$ being the rank of $a_{\lambda}$ and $b_{\mu}$ respectively; furthermore, let $\alpha_{0} \neq-\infty$ and $\beta_{0} \neq-\infty$. Then, according to the previous section, the characteristic sets

$$
S_{A}=\left\{\left(m_{1}^{\prime}, t_{1}^{\prime}\right), \cdots,\left(m_{f}^{\prime}, t_{f}^{\prime}\right)\right\}
$$

* The occurrence of $m_{\zeta}\left(m_{\zeta+1}\right)$ in the lemma is not to be interpreted as implying that $m_{\zeta}\left(m_{\zeta+1}\right)$ exists; for it is our purpose, as shown in the formulation of the lemma, to include also the cases $\zeta=0$, when $m_{0}$ does not exist, and $\zeta=h$, when $m_{\zeta+1}$ does not exist. If $m_{\zeta}\left(m_{\zeta+1}\right)$ does not exist, every relation rendered meaningless because of this non-existence is regarded as being fulfilled vacuously. Thus, if $\zeta=0, m_{0}$ is non-existent and $m_{0}>-1$ is regarded as true. On the other hand, every relation that has meaning requires proof. In the light of these remarks, similar situations below-and a few above-are to be interpreted. 
and

$$
S_{B}=\left\{\left(m_{1}^{\prime \prime}, t_{1}^{\prime \prime}\right), \cdots,\left(m_{g}^{\prime \prime}, t_{g}^{\prime \prime}\right)\right\}
$$

exist. The sum $S_{A}+S_{B}$ is defined (for $r \geqq 1$ and $s \geqq 1$ ) as a new set of number pairs

$$
S_{c}=\left\{\left(m_{1}, t_{1}\right), \cdots,\left(m_{h}, t_{h}\right)\right\},
$$

the $m$ 's and the $t$ 's being defined in the following manner:* The sequence $\left\{m_{1}, m_{2}, \cdots, m_{h}\right\}$ is obtained by arranging the (single) set of numbers $m_{\nu}^{\prime}\{\nu=1,2, \cdots, f\}$ and $m_{\nu}^{\prime \prime}\{\nu=1,2, \cdots, g\}$ in a decreasing sequence according to magnitude and discarding duplicates. With each $m$ associate the number $t=t_{\mu}^{\prime}$ or $t=t_{\nu}^{\prime \prime}$ that corresponds to it in either $S_{A}$ or $S_{B}$ if there is just one such correspondent; if there are two, $t_{\mu}^{\prime}$ in $S_{A}$ and $t_{\nu}^{\prime \prime}$ in $S_{B}$, associate with $m$ the number $t=t_{\mu}^{\prime}+t_{\nu}^{\prime \prime}$. Furthermore, $S_{A}+S_{B}$ is defined to be $S_{B}$ or $S_{A}$ according as $S_{A}=0$ (which is the case when $r=0$ ) or $S_{B}=0$ (which is the case when $s=0$ ).

From the definition of $S_{A}+S_{B}$ it follows that to every segment

$$
S_{c_{\zeta}}=\left\{\left(m_{1}, t_{1}\right), \cdots,\left(m_{\zeta}, t_{\zeta}\right)\right\}
$$

of $S_{G}$, where $\zeta=0,1,2, \cdots, h$, there correspond uniquely two segments

of $S_{A}$ and

$$
S_{\Delta_{\xi}}=\left\{\left(m_{1}^{\prime}, t_{1}^{\prime}\right), \cdots,\left(m_{\xi}^{\prime}, t_{\xi}^{\prime}\right)\right\}
$$

$$
S_{B_{\eta}}=\left\{\left(m_{1}^{\prime \prime}, t_{1}^{\prime \prime}\right), \cdots,\left(m_{\eta}^{\prime \prime}, t_{\eta}^{\prime \prime}\right)\right\}
$$

of $S_{B}$ such that $S_{A_{\xi}}+S_{B_{\eta}}=S_{C_{\zeta}}$. Two segments $S_{A_{\xi}}$ and $S_{B_{\eta}}$ thus determined are said to be "conjugate." There are thus in all $h+1$ pairs of conjugate segments.

Two conjugate segments $S_{A_{\xi}}$ and $S_{B_{\eta}}$ are characterized by the property that $m_{\xi}^{\prime}>m_{\eta+1}^{\prime \prime}$ and $m_{\eta}^{\prime \prime}>m_{\xi+1}^{\prime}$. (The null-segments and the segments $S_{A_{f}}=S_{A}$, $S_{B_{g}}=S_{B}$ share this property on account of our agreement regarding vacuous validity.)

It is evident that the $h+1$ pairs of conjugate segments may be so ordered, - and in only one way - that $\xi \leqq \xi^{\prime}$ and $\eta \leqq \eta^{\prime}$ (and furthermore, of course, either $\xi<\xi^{\prime}$ or $\left.\eta<\eta^{\prime}\right)$, whenever the pair $\left(S_{A_{\xi}}, S_{B_{\eta}}\right)$ precedes the pair $\left(S_{\boldsymbol{A}_{\xi^{\prime}}}, S_{B_{\eta^{\prime}}}\right)$. Then $\left(S_{A_{0}}, S_{B_{0}}\right)$ is the first pair, and $\left(S_{A_{f}}, S_{B_{g}}\right) \equiv\left(S_{A}, S_{B}\right)$, the last.

We introduce here, with regard to conjugate segments, a lemma that will simplify the proof of the "Theorem on the Product." This lemma gives us desirable information regarding the comparative values of the expressions $\alpha_{\lambda}+\beta_{\mu}-\sigma$ and $\alpha_{s_{\xi}^{\prime}}^{\prime}+\beta_{s_{\eta}}^{\prime \prime}($ see $\S 2)$. Here $s_{\xi}^{\prime}, s_{\eta}^{\prime \prime}$ stand, of course, for $\sum_{\nu=\xi}^{\nu} t_{\nu}^{\prime}$,

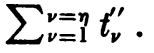

* The designation $S_{C}$ is not meant to imply here the existence of a parenthesis $C$ having $S_{C}$ as characteristic set. 
Lemma II. Let $A\left(\alpha_{0} \neq-\infty\right)$ and $B\left(\beta_{0} \neq-\infty\right)$ be two parentheses of $S_{;} S_{A_{\xi}}, S_{B_{\eta}}$, two conjugate segments of $S_{A}, S_{B} ;$ and $\lambda, \mu, \sigma$ integers such that $0 \leqq \lambda \leqq r, 0 \leqq \mu \leqq s$ and $0 \leqq \sigma$. Set

and

$$
\delta=\lambda+\mu+\sigma-\left(s_{\xi}^{\prime}+s_{\eta}^{\prime \prime}\right)
$$

Then

$$
\Delta=\alpha_{\lambda}+\beta_{\mu}-\sigma-\left(\alpha_{s_{\xi}^{\prime}}+\beta_{s_{\eta}^{\prime \prime}}\right) \text {. }
$$

(a) if $\delta>0$ and $m=\max \left(m_{\xi+1}^{\prime}, m_{\eta+1}^{\prime \prime}\right), \Delta \leqq \delta m$;

(b) if $\delta=0, m_{\xi}^{\prime}>-1, m_{\eta}^{\prime \prime}>-1$ and $[\lambda, \mu, \sigma] \neq$ 的, $\left.s_{\eta}^{\prime \prime}, 0\right], \Delta<0$.

Proof of $(a)$. On account of our agreement regarding vacuous validity, there is nothing to prove if both $m_{\xi+1}^{\prime}$ and $m_{\eta+1}^{\prime \prime}$ are non-existent.* On account of symmetry, we may then assume without losing generality that $m_{\xi+1}^{\prime}$ exists and $\geqq m_{\eta+1}^{\prime \prime}$. From Lemma I, we see that $\alpha_{\lambda}-\alpha_{\xi_{\xi}^{\prime}} \leqq\left(\lambda-s_{\xi}^{\prime}\right) m_{\xi+1}^{\prime}$; furthermore, since $S_{A_{\xi}}$ and $S_{B_{\eta}}$ are conjugate, $m_{\eta}^{\prime \prime}>m_{\xi+1}^{\prime} \geqq m_{\eta+1}^{\prime \prime}$ and therefore $\beta_{\mu}-\beta_{s_{\eta}^{\prime \prime}} \leqq\left(\mu-s_{\eta}^{\prime \prime}\right) m_{\xi+1}^{\prime}$; finally, since $-1 \leqq m_{\xi+1}^{\prime},-\sigma \leqq \sigma m_{\xi+1}^{\prime}$. Accordingly,

$$
\Delta \leqq\left(\lambda-s_{\xi}^{\prime}+\mu-s_{\eta}^{\prime \prime}+\sigma\right) m_{\xi+1}^{\prime}=\delta m .
$$

Proof of $(b)$. We suppose that $m_{\xi}^{\prime}$ and $m_{\eta}^{\prime \prime}$ both exist, the requisite modification of the argument being evident when this supposition is not fulfilled. Without loss of generality, we may assume, on account of symmetry, that $m_{\xi}^{\prime} \geqq m_{\eta}^{\prime \prime}$. We divide our discussion into two parts according as $\sigma>0$ or $\sigma=0$.

If $\sigma>0,-\sigma<\sigma m_{\eta}^{\prime \prime}$, since $-1<m_{\eta}^{\prime \prime}$ by hypothesis. Furthermore, since $S_{A_{\xi}}$ and $S_{B_{\eta}}$ are conjugate segments, $m_{\xi}^{\prime} \geqq m_{\eta}^{\prime \prime}>m_{\xi+1}^{\prime}$; hence (Lemma I)

$$
\alpha_{\lambda}-\alpha_{s_{\xi}^{\prime}} \leqq\left(\lambda-s_{\xi}^{\prime}\right) m_{\eta}^{\prime \prime} .
$$

Since, finally, $\beta_{\mu}-\beta_{s_{\eta}^{\prime \prime}} \leqq\left(\mu-s_{\eta}^{\prime \prime}\right) m_{\eta}^{\prime \prime}$, we have

$$
\Delta<\left(\lambda-s_{\xi}^{\prime}+\mu-s_{\eta}^{\prime \prime}+\sigma\right) m_{\eta}^{\prime \prime}=0 .
$$

If $\sigma=0$, it follows from $[\lambda, \mu, \sigma] \neq\left[s_{\xi}^{\prime}, s_{\eta}^{\prime \prime}, 0\right]$ and $\delta=0$ that either (1) $\lambda-s_{\xi}^{\prime}>0$ and $\mu-s_{\eta}^{\prime \prime}<0$ or (2) $\lambda-s_{\xi}^{\prime}<0$ and $\mu-s_{\eta}^{\prime \prime}>0$. In the first case, $m_{\xi+1}^{\prime}$ exists and (Lemma I, Remark)

$$
\alpha_{\lambda}-\alpha_{\xi_{\xi}^{\prime}} \leqq\left(\lambda-s_{\xi}^{\prime}\right) m_{\xi+1}^{\prime}<\left(\lambda-s_{\xi}^{\prime}\right) m_{\eta}^{\prime \prime} .
$$

Since, moreover, $\beta_{\mu}-\beta_{s_{\eta}^{\prime \prime}} \leqq\left(\mu-s_{\eta}^{\prime \prime}\right) m_{\eta}^{\prime \prime}$, it follows that

* As indicated in a previous footnote $(\$ 4)$, it is not presupposed in Lemma II that $m_{\xi}^{\prime}$, $m_{\xi+1}^{\prime}, m_{\eta}^{\prime \prime}, m_{\eta+1}^{\prime \prime}$ exist. It is our purpose to include in the application of the lemma the cases $\xi=0, \eta=0, \xi=f, \eta=g$. If $m_{\xi+1}^{\prime}$ exists but $m_{\eta+1}^{\prime \prime}$ does not, it is to be understood that $\max \left(m_{\xi+1}^{\prime}, m_{\eta+1}^{\prime \prime}\right)=m_{\xi+1}^{\prime}$; similarly, if $m_{\eta+1}^{\prime \prime}$ exists and $m_{\xi+1}^{\prime}$ does not. Since the relation $\Delta<0$ has meaning in $(b)$, even when $m_{\xi}^{\prime}$ or $m_{\eta}^{\prime \prime}$ or both are non-existent, it requires proof also in the latter cases. 


$$
\Delta<\left(\lambda-s_{\xi}^{\prime}+\mu-s_{\eta}^{\prime \prime}\right) m_{\eta}^{\prime \prime}=0 .
$$

In the second case, $\beta_{\mu}-\beta_{s_{\eta}}{ }^{\prime \prime}<\left(\mu-s_{\eta}^{\prime \prime}\right) m_{\eta}^{\prime \prime}$ (Lemma I, Remark). Since, moreover,

$$
\alpha_{\lambda}-\alpha_{s_{\xi}}^{\prime} \leqq\left(\lambda-s_{\xi}^{\prime}\right) m_{\xi}^{\prime} \leqq\left(\lambda-s_{\xi}^{\prime}\right) m_{\eta}^{\prime \prime}
$$

(on account of $\lambda-s_{\xi}^{\prime}<0$ and $m_{\eta}^{\prime \prime} \leqq m_{\xi}^{\prime}$ ), $\Delta<0$.

\section{The “THEOREM ON THE PRODUCT"*}

Theorem. If $A\left(\alpha_{0} \neq-\infty\right)$ and $B\left(\beta_{0} \neq-\infty\right)$ are two parentheses of $\mathfrak{S}$ and $C=A \cdot B$, then $S_{C}=S_{A}+S_{B}$.

Proof. $\quad C$ is a parenthesis of $\subseteq$ by virtue of $\S 2$. Moreover,

$$
\gamma_{0}=\alpha_{0}+\beta_{0} \text {. }
$$

For, if in (b) $\S 2$ we set $\nu=0$, it follows, on account of $0 \leqq \lambda, 0 \leqq \mu, 0 \leqq \sigma$, that $\lambda=0, \mu=0, \sigma=0$ for every $[\lambda, \mu, \sigma]_{\lambda+\mu+\sigma=\nu}$; hence there is no $[\lambda, \mu, \sigma]_{\lambda+\mu+\sigma=\nu} \neq=[\bar{\lambda}, \bar{\mu}, 0] \equiv[0,0,0]$. The hypothesis of (b) $\S 2$ is therefore fulfilled vacuously, and in consequence (1) holds. From (1) it follows that $\gamma_{0}$ is finite and accordingly that $S_{C}$ exists.

The proof that $S_{C}=S_{A}+S_{B}$ is effected by means of mathematical induction. We thus assume that it has already been established for the conjugate segments $S_{A_{\xi}}, S_{B_{\eta}}$ of $S_{A}, S_{B}$ that a segment $S_{C_{\zeta}}$ of $S_{C}$ exists such that

$$
S_{C_{\zeta}}=S_{A_{\xi}}+S_{B_{\eta}} \text {. }
$$

Since $S_{C_{0}}=S_{A_{0}}+S_{B_{0}}$ (each term in this equation being the null-set), the theorem will be completely proved, if, on the basis of the assumption (2), we show that $m_{\zeta+1}$ and $t_{\zeta+1}$ have values in accordance with the implications of $S_{C}=S_{A}+S_{B}$. (Compare the ordering of the $h+1$ pairs of conjugate segments, mentioned in $\S 5$.) This is what we shall do.

From (2) it follows, in accordance with the definition of the sum of two characteristic sets, that

$$
s_{\zeta}=s_{\xi}^{\prime}+s_{\eta}^{\prime \prime} \text {. }
$$

There is nothing to prove if neither $m_{\xi+1}^{\prime}$ nor $m_{\eta+1}^{\prime \prime}$ exists; for then, according to $(3)$ and $\$ 2$,

$$
s_{\zeta}=s_{f}^{\prime}+s_{g}^{\prime \prime}=r+s=n,
$$

and therefore $m_{\zeta+1}$ does not exist. Hence we may also assume, without loss of generality, that $m_{\xi}^{\prime}>-1$ and $m_{\eta}^{\prime \prime}>-1$. For suppose $m_{\xi}^{\prime}=-1$ (a similar argument will hold if $m_{\eta}^{\prime \prime}=-1$ ); then $m_{\xi+1}^{\prime}$ does not exist. Moreover, on account of the conjugacy of $S_{A_{\xi}}$ and $S_{B_{\eta}}$,

\footnotetext{
* This designation was suggested by Professor E. W. Davis.
} 


$$
-1=m_{\xi}^{\prime}>m_{\eta+1}^{\prime \prime} \geqq-1 \text {. }
$$

Hence $m_{\eta+1}^{\prime \prime}$ does not exist (or else we may conclude that $-1>-1$ ).* From $m_{\xi}^{\prime}>-1$ and $m_{\eta}^{\prime \prime}>-1$, it follows, however, by virtue of $1 d, \S 4$, and the relations (1) and (2) of the present section, that

$$
\gamma_{s_{\zeta}}=\alpha_{s_{\xi}^{\prime}}+\beta_{s_{\eta}^{\prime \prime}} \text {. }
$$

On account of symmetry, we may furthermore assume, without loss of generality, that $m_{\xi+1}^{\prime}$ exists and $\geqq m_{\eta+1}^{\prime \prime}$. We now divide our discussion (in natural manner) into the following three parts:

(a) $m_{\xi+1}^{\prime}=-1$. According to (a) Lemma II, if

$$
\nu=\lambda+\mu+\sigma>s_{\xi}^{\prime}+s_{\eta}^{\prime \prime}=s_{\zeta},
$$

we have, since $\delta=\nu-s_{\zeta}, \alpha_{s_{\xi}^{\prime}}^{\prime}+\beta_{s_{\eta}}^{\prime \prime}=\gamma_{s_{\zeta}}$, and $m=m_{\xi+1}^{\prime}=-1$ :

$$
\alpha_{\lambda}+\beta_{\mu}-\sigma \leqq \gamma_{s_{\zeta}}-\left(\nu-s_{\zeta}\right) \text {. }
$$

Hence, by virtue of $(a) \S 2, \gamma_{\nu} \leqq \gamma_{s_{\zeta}}-\left(\nu-s_{\zeta}\right)$ for every $\nu>s_{\zeta}$; i. e.,

$$
\frac{\gamma_{\nu}-\gamma_{s_{\zeta}}}{\nu-s_{\zeta}}\left\{\nu>s_{\zeta}\right\} \leqq-1 \text {. }
$$

According to the definition of " characteristic set," this shows that $m_{\zeta+1}=-1$ and $s_{\xi+1}=n=r+s$; that is, by (3), $t_{j+1}=t_{\xi+1}^{\prime}$ or $t_{\xi+1}^{\prime}+t_{\eta+1}^{\prime \prime}$ according as $m_{\eta+1}^{\prime \prime}$ is non-existent or existent (the value of $m_{\eta+1}^{\prime \prime}$ being -1 in the latter case).

(b) $m_{\xi+1}^{\prime}>-1$ and $>m_{\eta+1}^{\prime \prime}$ ( $m_{\eta+1}^{\prime \prime}$ being existent or non-existent). By means of $(a)$ Lemma II, we show, as in the case $m_{\xi+1}^{\prime}=-1$, that

$$
\frac{\gamma_{\nu}-\gamma_{s_{\zeta}}}{\nu-s_{\zeta}}\left\{\nu>s_{\zeta}\right\} \leqq m_{\xi+1}^{\prime} \text {. }
$$

Moreover, the segments $S_{A_{\xi+1}}$ and $S_{B_{\eta}}$ are conjugate; for, in addition to $m_{\xi+1}^{\prime}>m_{\eta+1}^{\prime \prime}$, we may conclude, on account of the assumed conjugacy of $S_{A_{\xi}}$ and $S_{B_{\eta}}$ and $1 b, \S 4$, that $m_{\eta}^{\prime \prime}>m_{\xi+1}^{\prime}>m_{\xi+2}^{\prime}$ (the latter relation holding vacuously when $m_{\xi+2}^{\prime}$ does not exist). It therefore follows from (b) Lemma II and $(b) \S 2$ that

$\gamma_{\nu}\left\{\nu=s_{\xi+1}^{\prime}+s_{\eta}^{\prime \prime}\right\}=\alpha_{s_{\xi+1}}^{\prime}+\beta_{s_{\eta}}^{\prime \prime}=\gamma_{s_{\zeta}}+t_{\xi+1}^{\prime} m_{\xi+1}^{\prime}=\gamma_{s_{\zeta}}+\left(\nu-s_{\zeta}\right) m_{\xi+1}^{\prime}$

whence

( $1 d, \S 4$ and (4) of present section),

$$
\frac{\gamma_{\nu}-\gamma_{s_{\zeta}}}{\nu-s_{\zeta}}\left\{\nu=s_{\xi+1}^{\prime}+s_{\eta}^{\prime \prime}\right\}=m_{\xi+1}^{\prime} \text {. }
$$

* If $m_{\xi}^{\prime}$ does not exist (as when $\xi=0$ ), the contradietion $-1>-1$ cannot be obtained even if $m_{\eta+1}^{\prime \prime}$ exists; but in that case, $m_{\xi}^{\prime}>-1$ holds vacuously. 
In view of the definition of " characteristic set," we may now conclude that $m_{\zeta+1}=m_{\xi+1}^{\prime}$. If neither $m_{\xi+2}^{\prime}$ nor $m_{\eta+1}^{\prime \prime}$ exists, $t_{\zeta+1}$ is evidently $t_{\xi+1}^{\prime}$ and there is nothing more to prove. We may therefore assume without losing generality that either $m_{\xi+2}^{\prime}$ or $m_{\eta+1}^{\prime \prime}$ exists; hence $m=\max \left(m_{\xi+2}^{\prime}, m_{\eta+1}^{\prime \prime}\right)$ exists. According to (a) Lemma II, we then see, in virtue of the conjugacy of $S_{\Delta_{\xi+1}}$ and $S_{B_{\eta}}$, that for $\nu=\lambda+\mu+\sigma>s_{\xi+1}^{\prime}+s_{\eta}^{\prime \prime}$, we have

But

$$
\alpha_{\lambda}+\beta_{\mu}-\sigma \leqq \alpha_{s_{\xi}+1}^{\prime}+\beta_{s_{\eta}^{\prime \prime}}^{\prime \prime}+\left(\nu-s_{\xi+1}^{\prime}-s_{\eta}^{\prime \prime}\right) m \text {. }
$$

$\alpha_{s_{\xi+1}^{\prime}}^{\prime}+\beta_{s_{\eta}^{\prime \prime}}^{\prime \prime}=\alpha_{s_{\xi}^{\prime}}^{\prime}+\beta_{s_{\eta}^{\prime \prime}}^{\prime \prime}+t_{\xi+1}^{\prime} m_{\xi+1}^{\prime}=\gamma_{s_{\xi}}+t_{\xi+1}^{\prime} m_{\xi+1}^{\prime}$

by (4); furthermore,

$$
\left(1 d, \S 4 \text {, since } m_{\xi+1}^{\prime}>-1\right),
$$

$$
\left(\nu-s_{\xi+1}^{\prime}-s_{\eta}^{\prime \prime}\right) m<\left(\nu-s_{\xi+1}^{\prime}-s_{\eta}^{\prime \prime}\right) m_{\xi+1}^{\prime},
$$

since $\nu-s_{\xi+1}^{\prime}-s_{\eta}^{\prime \prime}$ is positive and both $m_{\xi+2}^{\prime}$ and $m_{\eta+1}^{\prime \prime}$ are $<m_{\xi+1}^{\prime}$. Therefore

$$
\alpha_{\lambda}+\beta_{\mu}-\sigma<\gamma_{s_{\zeta}}+\left(\nu-s_{\xi}^{\prime}-s_{\eta}^{\prime \prime}\right) m_{\xi+1}^{\prime}=\gamma_{s_{\zeta}}+\left(\nu-s_{\zeta}\right) m_{\xi+1}^{\prime},
$$

by (3). Consequently, in accordance with $(a), \S(2)$,

whence

$$
\gamma_{\nu}<\gamma_{s_{\zeta}}+\left(\nu-s_{\zeta}\right) m_{\xi+1}^{\prime},
$$

$$
\frac{\gamma_{\nu}-\gamma_{s_{\zeta}}}{\nu-s_{\zeta}}\left\{\nu>s_{\xi+1}^{\prime}+s_{\eta}^{\prime \prime}\right\}<m_{\xi+1}^{\prime} \text {. }
$$

We now see that $s_{\zeta+1}=s_{\xi+1}^{\prime}+s_{\eta}^{\prime \prime}$ and hence $t_{\zeta+1}=t_{\xi+1}^{\prime}$.

(c) $m_{\xi+1}^{\prime}=m_{\eta+1}^{\prime \prime}>-1$. As in the previous case,

$$
\frac{\gamma_{\nu}-\gamma_{s_{\zeta}}}{\nu-s_{\zeta}}\left\{\nu>s_{\zeta}=s_{\xi}^{\prime}+s_{\eta}^{\prime \prime}\right\} \leqq m_{\xi+1}^{\prime} ;
$$

moreover, by means of (b) Lemma II for the conjugate segments $S_{\Lambda_{\xi+1}}$ and $S_{B_{\eta+1}}$ and $(b) \S 2$, it follows that

$$
\frac{\gamma_{\nu}-\gamma_{\delta_{\zeta}}}{\nu-s_{\zeta}}\left\{\nu=s_{\xi+1}^{\prime}+s_{\eta+1}^{\prime \prime}\right\}=m_{\xi+1}^{\prime} ;
$$

and finally, by means of (a) Lemma II for these segments and $(a) \S 2$ (as in the previous case), that

$$
\frac{\gamma_{\nu}-\gamma_{s_{\zeta}}}{\nu-s_{\zeta}}\left\{\nu>s_{\xi+1}^{\prime}+s_{\eta+1}^{\prime \prime}\right\}<m_{\xi+1}^{\prime} .
$$

From these relations we conclude that $m_{\xi+1}=m_{\xi+1}^{\prime}$ and that $t_{\xi+1}=t_{\xi+1}^{\prime}+t_{\eta+1}^{\prime \prime}$, thus completing the proof of the theorem. 
An immediate consequence of the "Theorem on the Product" is the

"Theorem on Common Divisors." If the parentheses $A\left(\alpha_{0} \neq-\infty\right)$ and $B\left(\beta_{0} \neq-\infty\right)$ are such that no $m_{\xi}^{\prime}\{\xi=1,2, \cdots, f\}$ is equal to an $m_{\eta}^{\prime \prime}\{\eta=1,2, \cdots, g\}$, they have no common factors of order $\geqq 1$.

For every $m$ of the characteristic set of a common factor is an $m$ of both $S_{A}$ and $S_{B}$.

\section{The " FACTORIzATION THEOREM" AND THE " CRITERION FOR IRREDUCIBILITY "}

If the parenthesis $C$ is such that $\gamma_{\nu}\{\nu=0,1, \cdots, n\}$ is an integer, which is the case in nearly all of the applications, $m_{\zeta}\{\zeta=1,2, \ldots, h\}$ is a rational number, being equal to $\left(\gamma_{s_{\zeta}}-\gamma_{s_{\zeta-1}}\right) / t_{\zeta}$ or -1 by $1 d, \S 4$. We then introduce the following

Definition of $\tau_{\zeta}$ and of $d_{\zeta}$. The number $\tau_{\zeta}$ is the (positive) denominator of $m_{\zeta}$ when $m_{\zeta}$ is written in its lowest terms, and $t_{\zeta}=d_{\zeta} \tau_{\zeta}$.

Suppose now that $C$ is decomposed into the product $A \cdot B$ of two parentheses, $A$ being subject to the further restriction that $\alpha_{\lambda}\{\lambda=0,1, \cdots, r\}$ is an integer. ( $B$ may be treated as we are going to deal with $A$.) Let $\tau_{\xi}^{\prime}$ and $d_{\xi}^{\prime}\{\xi=1,2, \cdots, f\}$ be defined for $A$ as $\tau_{\zeta}$ and $d_{\zeta}$ were for $C$. From the "Theorem on the Product," it follows that every $m_{\xi}^{\prime}$ is an $m_{\zeta}$; hence every $\tau_{\xi}^{\prime}$ is a $\tau_{\zeta}$. The "Theorem on the Product" tells us furthermore, that if $m_{\xi}^{\prime}=m_{\zeta}$, then $t_{\xi}^{\prime} \leqq t_{\zeta}$; hence $d_{\xi}^{\prime} \leqq d_{\zeta}$. Therefore,

$$
r=\sum \begin{aligned}
& \xi=f \\
& =1
\end{aligned} t_{\xi}^{\prime}=\sum \begin{aligned}
& \xi=f \\
& \xi=1
\end{aligned} d_{\xi}^{\prime} \tau_{\xi}^{\prime}=\sum \sum_{\xi=1}^{\xi=h} \nu_{\zeta} \tau_{\zeta},
$$

where $\nu_{\zeta}$ is an integer such that $0 \leqq \nu_{\zeta} \leqq d_{\zeta}$. We have thus proved the following

"Factorization Theorem." If $C$ is such that $\gamma_{\nu}\{\nu=0,1, \cdots, n\}$ is an integer (the value $-\infty$ being allowed except, of course, for $\gamma_{0}$ ), then the order of a factor $A$ of $C$, where $\alpha_{\lambda}\left\{\lambda^{\dot{1}}=0,1, \cdots, r\right\}$ is likewise an integer (or $\left.-\infty\right)$, is of the form

$$
r=\sum_{\zeta=1}^{h} \nu_{\zeta} \tau_{\zeta}
$$

where $\nu_{\zeta}$ is an integer such that $0 \leqq \nu_{\zeta} \leqq d_{\zeta}$ (and $\tau_{\zeta}, d_{\zeta}$ are defined as above).

In particular, the "Factorization Theorem " leads to the following

"CRiterion for IrReducibility." If the ranks of the elements of $\mathfrak{S}$ are all integers (or $-\infty)$, and $\left(\gamma_{n}-\gamma_{0}\right) / n$ is a fraction in its lowest terms $>-1$ and also $>\left(\gamma_{\nu}-\gamma_{0}\right) / \nu\{\nu=1,2, \cdots, n-1\}, C$ is irreducible.

For in this case, the characteristic set of $C$ consists of just one pair

$$
\left.\left(m_{1}, t_{1}\right)=\overline{\left(\gamma_{n}-\gamma_{0}\right.} / n, n\right)
$$


and moreover, since $\left(\gamma_{n}-\gamma_{0}\right) / n$ is in its lowest terms, $\tau_{1}=t_{1}=n$ and $d_{1}=1$. Hence, the order of a factor $A$ of $C$ is, according to the "Factorization Theorem," of the form

$$
r=\nu_{1} t_{1}=\nu_{1} n=0 \text { or } n .
$$

The difficulty of obtaining for $\mathfrak{T}_{-1}$ a more exhaustive criterion than the "Criterion for Irreducibility" may be judged from the following

Remarks. There cannot be a theorem for $\mathfrak{T}_{-1}$ that asserts the irreducibility of even a single parenthesis having more than one element in its characteristic set, unless the theorem asserts nothing for at least one of the situations of $\S 3$. For according to a result of Dumas, ${ }^{*}$ based on more general considerations of Hensel, $\dagger$ every parenthesis of Situation 2 having two or more elements in its characteristic set is reducible; that is, in Situation 2, $C$ is reducible unless either

or

$$
-1<\left(\gamma_{n}-\gamma_{0}\right) / n \geqq\left(\gamma_{\nu}-\gamma_{0}\right) / \nu \quad\{\nu=1,2, \cdots, n-1\}
$$

$$
S_{C}=\left\{\left(m_{1}, t_{1}\right)\right\}=\{(-1, n)\} .
$$

Furthermore, the "Criterion for Irreducibility" becomes false, if the condition that $\left(\gamma_{n}-\gamma_{0}\right) / n$ be a fraction in its lowest terms is not demanded. In fact, for every $n$ and $\gamma_{n}-\gamma_{0}$ that are not relatively prime, there is a $C$ belonging to Situation 1, for instance, that satisfies every other condition of our criterion and is nevertheless reducible, as the following example will show: Let $n=k d$ and $\gamma_{n}-\gamma_{0}=l d, k, l, d$ being integers such that $d>1$ (and $l \gtreqless 0)$. The parenthesis $C=y^{k d}-p^{-l d}$ of Situation 1 has the factor $y^{k}-p^{-l}$, although $\gamma_{0}=0, \gamma_{1}=-\infty, \gamma_{2}=-\infty, \cdots, \gamma_{n-1}=-\infty$, $\gamma_{n}=l d, \quad\left(\gamma_{n}-\gamma_{0}\right) / n=(l d / k d)>\left(\gamma_{\nu}-\gamma_{0}\right) / \nu \quad\{\nu=1,2, \cdots, n-1\}$. Finally, our criterion becomes false if the condition $\left(\gamma_{n}-\gamma_{0}\right) / n>-1$ is made milder to the extent of replacing 1 by a number $\epsilon>1$, however small $\epsilon-1$ may be. (If $\left(\gamma_{n}-\gamma_{0}\right) / n=-1, n$ must be 1 on account of the relative primeness of $n$ and $\gamma_{n}-\gamma_{0}$, and our criterion is trivial.) This is shown by the following example in Situation $6: \ddagger$ Let

$$
C=\frac{d^{n} y}{d x^{n}}+x^{-n} \frac{d y}{d x}-n x^{-n-1} y .
$$

Here $\gamma_{0}=0, \gamma_{1}=-\infty, \gamma_{2}=-\infty, \cdots, \gamma_{n-2}=-\infty, \gamma_{n-1}=-n$, $\gamma_{n}=-n-1 ; \quad\left(\gamma_{n}-\gamma_{0}\right) / n=-(n+1) / n>\left(\gamma_{\nu}-\gamma_{0}\right) / \nu \quad\{\nu=1,2$,

${ }^{*}$ L. c., p. 223 .

† Neue Grundlagen der Arithmetik, Journal für Mathematik, vol. 127 (1904), pp. 51-84, Section 4.

$\ddagger$ It will appear in the next section that no such example is possible for Situations 1, 2, $3,4,5,8$, or 9 of $\S 3$, since these situations (as will appear) are instances of the Theory $\mathfrak{T}_{-\infty}$. As a consequence, the "Criterion for Irreducibility" holds for these situations if $\left(\gamma_{n}-\gamma_{0}\right) / n>-1$ is replaced by $\left(\gamma_{n}-\gamma_{0}\right) / n>-\infty$. 
$\cdots, n-1\}$. However small $\epsilon-1$ may be, $n$ may be so chosen that

Still, $C=A \cdot B$, where

$$
\frac{\gamma_{n}-\gamma_{0}}{n}>-\epsilon \text {. }
$$

$$
A=d y / d x \quad \text { and } \quad B=d^{n-1} y / d x^{n-1}+x^{-n} y .
$$

Clearly the "Criterion for Irreducibility" is not improved by replacing $\left(\gamma_{n}-\gamma_{0}\right) / n>\left(\gamma_{\nu}-\gamma_{0}\right) / \nu$ by $\left(\gamma_{n}-\gamma_{0}\right) / n \geqq\left(\gamma_{\nu}-\gamma_{0}\right) / \nu$; for the former condition is a consequence of the latter and the condition that $\left(\gamma_{n}-\gamma_{0}\right) / n$ is a fraction in its lowest terms.

\section{The abstKaCt THEORY $\mathfrak{T}_{e}$}

In his $1900 \mathrm{Mathematische} \mathrm{Annalen} \mathrm{paper,} \mathrm{Ueber} \mathrm{die} \mathrm{Irre-}$ ductibilität algebraischer Functionalgleichungen, Koenigsberger deals with a situation, which, if slightly modified to conform to our point of view, does not fall under the theory $\mathfrak{T}_{1}$. This situation, which we designate in accordance with our previous numbering as Situation 10, is essentially* as follows:

Situation 10. $\subseteq$ consists of the collectivity of fractions $e=e^{\prime} / e^{\prime \prime}$, where $e^{\prime}$ and $e^{\prime \prime}$ are power series in $x-\theta\left(=x_{1}\right.$, cf. Situation 5) with arbitrary complex coefficients, $\theta$ being constant with reference to $x$. As in Situation 5, the rank of $e(\neq 0)$ is defined as $d^{\prime \prime}-d^{\prime}$, where $d^{\prime}$ and $d^{\prime \prime}$ represent the lowest degrees (in the usual sense) in $x-\theta=x_{1}$ of a non-zero term of $e^{\prime}$ and $e^{\prime \prime}$ respectively; the rank of 0 is $-\infty$. The parenthesis $\left(e_{0}, e_{1}, \cdots, e_{m}\right)$ is the linear homogeneous differential expression

$$
e_{0}(x) \frac{d^{m} y}{d x^{m}}+e_{1}(x) \frac{d^{m-1} y}{d x^{m-1}}+\cdots+e_{m}(x) y
$$

The product of two parentheses is defined as in Situation 6 .

If we compare Situation 10 with the illustrative example of $\S 1$ (that is, with Situation 6 ), we see it to be no longer true, as it was there, that the rank of $d e(x) / d x$ is always less than or equal to the rank of $e(x)$; the rank of $x-\theta$, for example, is -1 , while the rank of $d(x-\theta) / d x$ is 0 . It is true, however, as may be verified in elementary fashion, that the rank of $d e(x) / d x$ is at most 1 more than the rank of $e(x)$. This new state of affairs requires a corresponding modification of the abstract theory $\mathfrak{T}_{-1}$. A natural modification leads to a theory, called-with propriety in the light of the definition of $\mathfrak{T}_{\mathrm{e}}$ below-

The Abstract Theory $\mathfrak{T}_{+1}$. Modify the Theory $\mathfrak{T}_{-1}$ by replacing in (a) and $(b), \S 2$, the expression $\alpha_{\lambda}+\beta_{\mu}-\sigma$ by $\alpha_{\lambda}+\beta_{\mu}+\sigma$, leaving everything else unaltered; the resulting theory is $\mathfrak{T}_{+1}$.

\footnotetext{
* Situation 10 is slightly more general than Koenigsberger's situation.
} 
The reader may easily verify that Situation 10 is an instance of the Theory $\mathfrak{T}_{+1}$.

Again, we proceed to show how another theory, properly called $\mathfrak{I}_{-\infty}$, naturally arises. It is easy to see that most of the situations of $\S 3$ satisfy more restrictive conditions than $(a)$ and $(b)$ of $\S 2$. In fact, the reader may verify that Situations $1,2,3,4,5,8$, and 9 actually satisfy the following conditions:

(a) $\gamma_{\nu} \leqq \max \left(\alpha_{\lambda}+\beta_{\mu}\right),\{0 \leqq \lambda \leqq r, 0 \leqq \mu \leqq s\}$.

(b) $\gamma_{\nu}=\alpha_{\bar{\lambda}}+\beta_{\bar{\mu}}\{\bar{\lambda}+\bar{\mu}=\nu\}$ if $\alpha_{\bar{\lambda}}+\beta_{\bar{\mu}}>\alpha_{\lambda}+\beta_{\mu}\{\lambda+\mu=\nu\}$ for every $[\lambda, \mu]$ 来 $[\bar{\lambda}, \bar{\mu}],\{0 \leqq \lambda \leqq r, 0 \leqq \mu \leqq s\}$.

It is evident, if we agree that $-\infty \cdot 0=0$, that these new conditions (a) and $(b)$ are equivalent to the following:

(a) $\gamma_{\nu} \leqq \max _{\lambda+\mu+\sigma=\nu}\left(\alpha_{\lambda}+\beta_{\mu} \neg \infty \cdot \sigma\right),\{0 \leqq \lambda \leqq r, 0 \leqq \mu \leqq s, 0 \leqq \sigma\}$.

(b) $\gamma_{\nu}=\alpha_{\bar{\lambda}}+\beta_{\bar{\mu}}\{\bar{\lambda}+\bar{\mu}=\nu\}$ if $\alpha_{\bar{\lambda}}+\beta_{\bar{\mu}}>\alpha_{\lambda}+\beta_{\mu}-\infty \cdot \sigma\{\lambda+\mu+\sigma$ $=\nu\}$ for every $[\lambda, \mu, \sigma] \neq[\bar{\lambda}, \bar{\mu}, 0],\{0 \leqq \lambda \leqq r, 0 \leqq \mu \leqq s, 0 \leqq \sigma\}$.

We thus arrive at

The Abstract Theory $\mathfrak{T}_{-\infty}$. Modify the Theory $\mathfrak{T}_{-1}$ by replacing in (a) and (b), § 2, the expression $\alpha_{\lambda}+\beta_{\mu}-\sigma$ by $\alpha_{\lambda}+\beta_{\mu}-\infty \cdot \sigma$ and agree that $-\infty \cdot 0=0$; the resulting theory is $\mathfrak{T}_{-\infty}$.

Situations 1-5 incl., 8 and 9 are instances of the Theory $\mathfrak{I}_{-\infty}$.

The considerations above lead to the idea of

The Abstract Theory $\mathfrak{T}_{\boldsymbol{e}}$ FOr eVery Real Number $\epsilon$ (and for $\epsilon=-\infty$ ). This is obtained from $\mathfrak{I}_{-1}$ by replacing in $(a)$ and $(b), \S 2$, the expression

$$
\alpha_{\lambda}+\beta_{\mu}-\sigma \text { by } \alpha_{\lambda}+\beta_{\mu}+\epsilon \sigma .
$$

It is evident that the Theory $\mathfrak{I}_{\mathrm{e}}$ is a $\mathfrak{T}_{\mathrm{\epsilon}^{\prime}}$ for $\epsilon^{\prime}>\epsilon$.

In the Theory $\mathfrak{T}_{e}$, "characteristic set," the "sum of two characteristic sets," " conjugate segments" and the numbers $\tau_{\zeta}$ and $d_{\zeta}$ of $\S 7$ may be defined similarly as in the Theory $\mathfrak{T}_{-1}$.

Thus, the "characteristic set" of $C$ in the Theory $\mathfrak{T}_{\mathbb{c}}$ is defined as follows:

$$
\begin{aligned}
m_{\zeta+1} & =\max _{\nu>s_{\zeta}}\left(\frac{\gamma_{\nu}-\gamma_{s_{\zeta}}}{\nu-s_{\zeta}}, \epsilon\right) . \\
s_{\zeta+1} & =\text { last } \nu>s_{\zeta} \text { such that } \frac{\gamma_{\nu}-\gamma_{s_{\zeta}}}{\nu-s_{\zeta}}=m_{\zeta+1} \text {, if } m_{\zeta+1}>\epsilon ; \text { and } \\
s_{\zeta+1} & =n \text {, if } m_{\zeta+1}=\epsilon .
\end{aligned}
$$

It is readily seen that the relations $1, \S 4$, hold in $\mathfrak{T}_{\varepsilon}$ if the last relation of (b) is changed to $m_{h} \geqq \epsilon$ and in $(d) m_{\zeta}>-1$ is changed to $m_{\zeta}>\epsilon$. Likewise, Lemma I holds if $m_{\zeta}>-1$ is replaced by $m_{\zeta}>\epsilon$, and the proof is precisely analogous. 
The "sum of two characteristic sets" and "conjugate segments" are defined in $\mathfrak{I}_{\mathrm{e}}$ precisely as in $\S 5$. Lemma II holds in $\mathfrak{T}_{\mathrm{e}}$ if $\Delta$ is set equal to

$$
\alpha_{\lambda}+\beta_{\mu}+\epsilon \sigma-\left(\alpha_{s_{\xi}^{\prime}}^{\prime}+\beta_{s_{\eta}}^{\prime \prime}\right),
$$

and in (b) $m_{\xi}^{\prime}>-1, m_{\eta}^{\prime \prime}>-1$ are replaced by $m_{\xi}^{\prime}>\epsilon, m_{\eta}^{\prime \prime}>\epsilon$, and the proof is precisely analogous.

From these considerations, it is evident that

The "Theorem on the Product" and the "Theorem on Common Divisors" hold for $\mathfrak{T}_{\mathrm{e}}$, and the proof is entirely analogous to that of $\S 6$.

In trying to carry over the results of $\S 7$ to the Theory $\mathfrak{T}_{e}$, we at first note a slight divergence; for even if the parenthesis $C$ is such that $\gamma_{\nu}\{\nu=0,1$, $\cdots, n\}$ is an integer, $m_{\zeta}$ need not be rational if equal to $\epsilon$. The divergence disappears, however, and in natural fashion, if we define $\tau_{\zeta}$ to be equal to +1 in case $m_{\zeta}=\epsilon$, whether $\epsilon$ is rational or not.* In other cases $\left(m_{\zeta}>\epsilon\right), \tau_{\zeta}$ is defined as in $\S 7$ and $d_{\zeta}$ (as there) always by the equation $t_{\zeta}=d_{\zeta} \tau_{\zeta}$. It then appears that

The "Factorization Theorem" holds in $\mathfrak{T}_{\mathrm{e}}$ and

The "Criterion for Irreducibility" holds in $\mathfrak{T}_{\mathrm{e}}$ if the relation

is replaced by the relation

$$
\left(\gamma_{n}-\gamma_{0}\right) / n>-1
$$

$$
\left(\gamma_{n}-\gamma_{0}\right) / n>\epsilon .
$$

The remarks at the close of $\S 7$ show the difficulty of obtaining a more exhaustive criterion for irreducibility in $\mathfrak{T}_{\mathfrak{e}}$.

ApPendix. Connections with the literature; concrete EXamples

(A) The Schoenemann-Eisenstein theorem says that

$$
C=y^{n}+i_{1} p y^{n-1}+i_{2} p y^{n-2}+\cdots+i_{n-1} p y+i_{n} p,
$$

where the $i$ 's are integers and $i_{n}$ not divisible by the prime integer $p$, is irreducible in the domain of rational numbers. This theorem is a special case of the "Criterion for Irreducibility" in the Theory $\mathfrak{I}_{-\infty}$ (or in $\mathfrak{T}_{\epsilon}$ for $\epsilon<-\frac{1}{2}$ ), when interpreted for Situation $1, \S 3$. For, $C=\left(c_{0}, c_{1}, \cdots, c_{n}\right)$, where $c_{0}=1, c_{1}=i_{1} p, \cdots, c_{n}=i_{n} p$; hence $\gamma_{0}=0, \gamma_{1} \leqq-1, \gamma_{2} \leqq-1, \cdots$, $\gamma_{n-1} \leqq-1, \gamma_{n}=-1$. Therefore,

* The reader will remember that in case $m_{\zeta}=\epsilon$, we have only an inequality

$$
\left(\gamma_{s_{\zeta}}-\gamma_{s_{\zeta-1}} \leqq m_{\zeta} t_{\zeta}\right)
$$

for $\gamma_{\iota}$ instead of the usual equality. 


$$
\left(\gamma_{n}-\gamma_{0}\right) / n=-1 / n>-\infty, \quad>\left(\gamma_{\nu}-\gamma_{0}\right) / \nu \quad\{\nu=1,2, \cdots, n-1\}
$$

and is in its lowest terms.*

The irreducibility of $y^{p-1}+y^{p-2}+\cdots+y+1 \quad(p=$ a prime integer $)$ in the domain of rational numbers is a direct consequence of the SchoenemannEisenstein theorem. $\dagger$

(B) The Floquet Theorem $\ddagger$ says that the linear homogeneous differential expression

$$
\begin{aligned}
C=(x-\theta)^{n+1} p_{0}(x) \frac{d^{n} y}{d x^{n}}+(x-\theta)^{n-1+\mu_{1}} p_{1}(x) \frac{d^{n-1} y}{d x^{n-1}}+\cdots \\
+(x-\theta)^{1+\mu_{n-1}} p_{n-1}(x) \frac{d y}{d x}+p_{n}(x) y
\end{aligned}
$$

is irreducible under the following conditions:

1. $C$ is said to be irreducible, if it is not expressible as a product of two linear homogeneous differential expressions of order $\geqq 1$, whose coefficients are power series in $x-\theta$ with arbitrary complex numerical coefficients.

2. $\mu_{1}, \mu_{2}, \cdots, \mu_{n-1}$ are all $\geqq 1$.

3. The $p$ 's are power series in $x-\theta$ such that $p_{0}(\theta), p_{1}(\theta), \cdots, p_{n}(\theta)$ are all $\neq 0$.

The theorem is a special case of the "Criterion for Irreducibility" in the Theory $\mathfrak{I}_{+1}$, when interpreted for Situation $10, \S 8$. For, we have

Hence

$$
\begin{gathered}
\gamma_{0}=-(n+1), \quad \gamma_{1}=-\left(n-1+\mu_{1}\right), \quad \cdots, \\
\gamma_{n-1}=-\left(1+\mu_{n-1}\right), \quad \gamma_{n}=0 .
\end{gathered}
$$

$\left(\gamma_{\nu}-\gamma_{0}\right) / \nu\{\nu=1,2, \cdots, n-1\}=\left(\nu+1-\mu_{\nu}\right) / \nu \leqq 1$

a fraction in its lowest terms.

$$
<(n+1) / n=\left(\gamma_{n}-\gamma_{0}\right) / n,
$$

(C) Koenigsberger, Journal für Mathematik, vol. 115, pp. 53-78. Every result of this paper (pertaining exclusively to the reducibility of expressions) falls under our theory. We select several examples.\$

(a) P. 55. "Wenn in einer algebraischen Gleichung

$$
C=c_{0}(x) y^{n}+c_{1}(x) y^{n-1}+\cdots+c_{n-1}(x) y+c_{n}(x)=0,
$$

${ }^{*}$ By noting that $C$ is irreducible when and only when $\left(c_{n}, c_{n-1}, \cdots, c_{0}\right)$ is irreduciblethis is, of course, not always so in the abstract theory-we may show that the SchoenemannEisenstein theorem is a consequence of the "Criterion for Irreducibility" in a $\mathfrak{T}_{\epsilon}, \epsilon \leqq 0$, interpreted for Situation 1, Section 3. A similar remark applies to other examples of the appendix.

$\dagger$ Eisenstein, 1. c.

¥ Contained in Koenigsberger, M a th e m a t i s c h e A n n a l e n, vol. 53 (1900), p. 67

$\S$ In the quoted theorems of the appendix, slight deviations from the original are made when greater harmony in statement with the rest of this paper may be thereby secured. 
in welcher $c_{0}(x), c_{1}(x), \cdots, c_{n}(x)$ ganze Functionen* von $x$ bedeuten, alle Coefficienten mit Ausnahme des ersten durch einen Linearfactor $x-\theta$, der letzte aber nicht durch $(x-\theta)^{2}$ theilbar ist, so ist die Gleichung irreductibel."

This theorem $\dagger$ is a special case of the "Criterion for Irreducibility," $\mathfrak{T}_{-\infty}$, Situation $5, \S 3, k$ being taken equal to 1 , and $x_{1}$ set equal to $x-\theta$. It is seen that $\gamma_{0}=0, \gamma_{1} \leqq-1, \gamma_{2} \leqq-1, \cdots, \gamma_{n-1} \leqq-1, \gamma_{n}=1$, as in $(A)$.

(b) P. 63. Theorem. Every polynomial of the form

$$
\begin{aligned}
C=p_{0}(x) y^{n}+(x-\theta)^{E(i / n)+1} & p_{1}(x) y^{n-1}+(x-\theta)^{E(2 i / n)+1} p_{2}(x) y^{n-2} \\
& +\cdots+(x-\theta)^{E[(n-1) / / n]+1} p_{n-1}(x)+(x-\theta)^{i} p_{n}(x)
\end{aligned}
$$

is irreducible under the following conditions:

1. The $p$ 's are polynomials in $x$, and the domain of rationality is the set of rational functions of $x$ with arbitrary complex coefficients; $p_{0}(\theta) \neq 0$ and $p_{n}(\theta) \neq 0$.

2. $i$ is a positive integer relatively prime to $n ; E(t)$ signifies, as usual, the largest integer $\leqq t$.

The theorem is a special case of the "Criterion for Irreducibility" in $\mathfrak{I}_{-\infty}$, Situation $5, k=1, x_{1}=x-\theta$. For, if $\gamma_{v}$ represents the rank of the coefficient of $y^{n-\nu}$, we have $\gamma_{0}=0, \gamma_{1} \leqq-E(i / n)-1, \gamma_{2} \leqq-E(2 i / n)-1$, $\cdots, \gamma_{n-1} \leqq-E(\overline{n-1} i / n)-1, \gamma_{n}=-i ; \quad\left(\gamma_{n}-\gamma_{0}\right) / n=-i / n, \quad$ a fraction in its lowest terms and furthermore $\left(\gamma_{n}-\gamma_{0}\right) / n>\left(\gamma_{\nu}-\gamma_{0}\right) / \nu$ $\{\nu=1,2, \cdots, n-1\}$.

(c) P. 77. "Jede algebraische Gleichung $n$-ten Grades von der Form

$$
\begin{aligned}
& C=i_{0} y^{n}+p^{\kappa_{\mu}^{(1)}+(1 / \mu)} q_{\nu}^{\kappa_{\nu}^{(1)}+(1 / \nu)} i_{1} y^{n-1}+p_{\mu}^{\kappa_{\mu}^{(2)}+(2 / \mu)} q_{\nu}^{\alpha_{\nu}^{(2)}+(2 / \nu)} i_{2} y^{n-2}+\cdots
\end{aligned}
$$

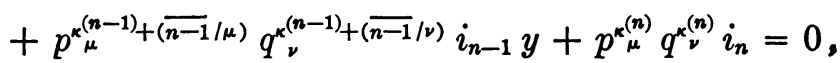

in welcher $n=\mu \cdot \nu, \mu$, und $\nu$ relative Primzahlen, $p$ und $q$ zwei verschiedene Primzahlen, $i_{0}, i_{1}, \cdots, i_{n}$ beliebige ganze Zahlen, von denen nur $i_{0}$ und $i_{n}$ weder durch $p$ noch durch $q$ theilbar sind,

$$
\kappa_{\mu}^{(\rho)}=E(\overline{\rho-1} / \mu)+1, \quad \kappa_{\nu}^{(\rho)}=E(\overline{\rho-1} / \nu)+1,
$$

also $\kappa_{\mu}^{(n)}=\nu, \kappa_{\nu}^{(n)}=\mu$ ist, endlich das Symbol $(\rho / \sigma)$ die Einheit oder Null bedeutet, je nachdem $\rho$ durch $\sigma$ theilbar oder nicht theilbar, ist irreductibel."

The theorem follows from the "Factorization Theorem," $\mathfrak{T}_{-\infty}$, Situation 1. For, let $\gamma_{\nu}$ be the rank of the coefficient of $y^{n-\nu}$ with respect to the prime $p$;

* By "ganze Functionen," Koenigsberger means polynomials; the theorem is also true, however, when the $c$ 's are power series in $x-\theta$.

† The domain of rationality is not given explicitly in the statement of the theorem; however, the subsequent statement that the theorem comes under Situation 5 supplies the deficiency. A similar remark applies to other examples of the appendix. 
$\gamma_{\nu}^{\prime}$, the rank of the same coefficient with respect to the prime $q$. We then have:

$$
\begin{aligned}
\gamma_{0}=0, \quad \gamma_{1} \leqq-\kappa_{\mu}^{(1)}-(1 / \mu), & \gamma_{2} \leqq-\kappa_{\mu}^{(2)}-(2 / \mu), \cdots, \\
\gamma_{n-1} \leqq-\kappa_{\mu}^{(n-1)}-(\overline{n-1} / \mu), & \gamma_{n}=-\kappa_{\mu}^{(n)}=-\nu ; \\
\left(\gamma_{n}-\gamma_{0}\right) / n=-\nu / n \geqq\left(\gamma_{\nu}-\gamma_{0}\right) / \nu & \{\nu=1,2, \cdots, n-1\} .
\end{aligned}
$$

The characteristic set (with respect to $p$ ) consists of the single element $\left(m_{1}, t_{1}\right)=(-\nu / n, n) ; \tau_{1}=\mu$ and $d_{1}=\nu$. Hence, according to the "Factorization Theorem," the order $r$ of a factor of $C$ is of the form $r=i \mu$, where $i$ is an integer. Similarly, by reasoning with the ranks $\gamma_{\nu}^{\prime}$ of the coefficients with respect to $q$, we conclude that $r=j \nu$, where $j$ is an integer. Since $\mu$ and $\nu$ are relatively prime, $r$ must be of the form $\kappa \mu \nu=\kappa n$. Hence, $r=0$ or $n$; that is, $C$ is irreducible.

Example.

$C=i_{0} y^{6}+p q i_{1} y^{5}+p^{2} q i_{2} y^{4}+p^{2} q^{2} i_{3} y^{3}+p^{3} \cdot q^{2} i_{4} y^{2}$

$i_{0}$ and $i_{6}$ being divisible by neither $p$ nor $q$.

$$
+p^{3} q^{2} i_{5} y+p^{3} q^{2} i_{6}=0,
$$

It is evident that a more general theorem may be at once obtained by taking $k$ primes ( $k$ arbitrary) instead of the two primes $p$ and $q$.

(D) Netto, Mathematische Annalen, vol. 48, pp. 81-88. All the results may be treated from our point of view. We select several examples.

(a) Pp. 82-83. Theorem. The polynomial

$$
y^{2 n+1}+i_{1} \cdot p y^{2 n}+\cdots+i_{n} p y^{n+1}+i_{n+1} p^{2} y^{n}+\cdots+i_{2 n+1} p^{2},
$$

where $p$ is a prime, $i_{\nu}\{\nu=1,2, \cdots, 2 n+1\}$ an integer and $i_{2 n+1}$ not divisible by $p$, is irreducible.

The theorem is a special case of the "Criterion for Irreducibility?" $\mathfrak{I}_{-\infty}$, Situation 1.

(b) P. 85. "Wenn im Polynome

$$
C=y^{n}+c_{1} y^{n-1}+c_{2} y^{n-2}+\cdots+c_{n-1} y+c_{n}
$$

alle Coefficienten $c_{\nu}$ durch $p$ theilbar sind, $c_{n-1}$ aber durch keine höhere Potenz von $p$ als die erste, dann ist es entweder irreductibel oder es zerfällt in der Weise, dass es einen Factor des Grades 1 und einen anderen irreductiblen Factor des Grades $n-1$ besitzt."

The theorem is a special case of the "Factorization Theorem," $\mathfrak{T}_{-\infty}$, Situation 1. For

$$
\gamma_{0}=0, \quad \gamma_{1} \leqq-1, \quad \cdots, \quad \gamma_{n-1}=-1, \quad \gamma_{n} \leqq-1 ;
$$




$$
\begin{aligned}
\left(\gamma_{n}-\gamma_{0}\right) / n \gtreqless\left(\gamma_{n-1}-\gamma_{0}\right) /(n-1)>\left(\gamma_{\nu}-\gamma_{0}\right) / \nu & \\
& \{\nu=n-2, n-3, \cdots, 1\} .
\end{aligned}
$$

It is hence evident that the first pair $\left(m_{1}, t_{1}\right)$ of the characteristic set of $C$ is either $(-1 /(n-1), n-1)$-and then $t_{2}$ of the second pair equals 1 -or $(-1 / n, n)$. Therefore, according to the "Factorization Theorem," the order of a factor of $C$ is of the form $i(n-1)+j$ or $i n$, where $i$ and $j$ have the values 0 or 1 . The theorem is thus proved.

(E) Perron, Mathe mat is che Annale n, vol. 60, pp. 448-458. Every result falls under our theory. We select several examples.

(a) P. 452. "Die algebraische Gleichung

$$
\begin{aligned}
& C=y^{n}+p_{1}^{\left.E \varepsilon_{1} / n\right)+1} p_{2}^{E\left(e_{2} / n\right)+1} \cdots p_{k}^{E\left(\varepsilon_{k} / n\right)+1} i_{1} y^{n-1}+p_{1}^{E\left(2 \varepsilon_{1} / n\right)+1} \cdots p_{k}^{\left.E 2 \varepsilon_{k} / n\right)-1} i_{2} y^{n-2} \\
& +\cdots+p_{1}^{R\left(n-1 e_{1} / n\right)+1} \cdots p_{k}^{E\left[(n-1) e_{k} / n\right]+1} i_{n-1} y+p_{1}^{e_{1}} \cdots p_{k}^{e_{k}} i_{n}=0,
\end{aligned}
$$

wo $i_{1}, \cdots, i_{n}$ beliebige ganze rationale Zahlen bedeuten, deren letzte zu den Primzahlen $p_{1}, p_{2}, \cdots, p_{k}$ prim ist, wo ferner die $k+1$ Zahlen $n, e_{1}, e_{2}$, $\cdots, e_{k}$ keinen gemeinsamen Teiler haben, ist irreduzibel." (The $e$ 's are $>0$.)

The theorem is a special case of the "Factorization Theorem," $\mathfrak{T}_{-\infty}$, Situation 1. For, let $\gamma_{\nu, \sigma}\{\nu=0,1, \cdots, n ; \sigma=1,2, \cdots, k\}$ be the rank of the coefficient of $y^{n-\nu}$ with respect to the prime $p_{\sigma}$. Then

$$
\begin{gathered}
\gamma_{0, \sigma}=0, \quad \gamma_{1, \sigma} \leqq-\left(e_{\sigma} / n\right)-\theta_{1, \sigma}, \quad \gamma_{2, \sigma} \leqq-\left(2 e_{\sigma} / n\right)-\theta_{2, \sigma}, \cdots, \\
\gamma_{n-1, \sigma} \leqq-\left((n-1) e_{\sigma} / n\right)-\theta_{n-1, \sigma}, \quad \gamma_{n, \sigma}=-e_{\sigma},
\end{gathered}
$$

where the $\theta$ 's lie between 0 (exclusive) and 1 (inclusive);

$$
\left(\gamma_{n, \sigma}-\gamma_{0, \sigma}\right) / n=-\left(e_{\sigma} / n\right)>\left(\gamma_{\nu, \sigma}-\gamma_{0, \sigma}\right) / \nu \quad\{\nu=1,2, \cdots, n-1\} .
$$

It then follows from the "Factorization Theorem," the ranks of the coefficients being taken with respect to $p_{\sigma}$, that the characteristic set consists of the single element $\left(-e_{\sigma} / n, n\right)$. Hence, the order $r$ of a factor of $C$ has the form $\left(i n /\left[n, e_{\sigma}\right]\right)$, where $i$ is an integer and $\left[n, e_{\sigma}\right]=$ G.C.D. of $n$ and $e_{\sigma}$. Therefore $r\left[n, e_{\sigma}\right]$ is a multiple of $n$ for $\sigma=1,2, \cdots, k$. Since the G.C.D. of the $k$ numbers $\left[n, e_{\sigma}\right.$ ] is 1 by hypothesis, there exist $k$ integers $j_{\sigma}$ such that $\sum_{\sigma=1}^{\sigma=k} j_{\sigma}\left[n, e_{\sigma}\right]=1$. Hence $r=\sum_{\sigma=1}^{\sigma=k} j_{\sigma} r\left[n, e_{\sigma}\right]$ is a multiple of $n$; that is, $r=0$ or $n$.

(b) Pp. 455-456. “Das Polynom

$$
y^{n}+i_{1} y^{n-1}+\cdots+i_{k} y^{n-k}+p i_{k+1} y^{n-k-1}+\cdots+p i_{n},
$$

oder allgemeiner 


$$
\begin{aligned}
C=y^{n}+i_{1} y^{n-1}+\cdots+i_{k} y^{n-k}+p^{E(e / \overline{n-k})+1} i_{k+1} y^{n-k-1}+\cdots \\
+p^{E[(n-k-1) e / \overline{n-k}]+1} i_{n-1} y+p^{e} i_{n},
\end{aligned}
$$

wo $i_{k}$ und $i_{n}$ durch die Primzahl $p$ nicht teilbar sind, und wo $e$ zu $n-k$ relativ prim ist, enthält mindestens einen irreduzibeln Faktor, dessen Grad $\geqq n-k$ ist."

A special case of the "Factorization Theorem," $\mathfrak{T}_{-\infty}$, Situation 1.

(F) Koenigsberger, M a thematis che Annalen, vol. 53, pp. 49-80. Every result (within our present field of investigation) is a special case of the "Factorization Theorem" either in $\mathfrak{T}_{+1}$ for Situation 10 or in $\mathfrak{T}_{-\infty}$ for Situation $5, k=1, x_{1}=x-\theta$. We select several illustrations.

(a) P. 69. "Die lineare Differentialgleichung

$$
\begin{aligned}
C=(x-\theta)^{n+2} p_{0}(x) \frac{d^{n} y}{d x^{n}}+(x & -\theta)^{n-1+\mu_{1}} p_{1}(x) \frac{d^{n-1} y}{d x^{n-1}}+\cdots \\
& +(x-\theta)^{1+\mu_{n-1}} p_{n-1}(x) \frac{d y}{d x}+p_{n}(x) y=0,
\end{aligned}
$$

in welcher $\mu_{1}, \mu_{2}, \cdots, \mu_{n-1} \geqq 2$ sind, ist, wenn die Ordnung $n$ derselben ungerade, stets irreductibel; ist die Ordnung jedoch gerade, so kann sie nur mit einer gleichartigen linearen Differentialgleichung $n / 2$ ter Ordnung die sämmtlichen Integrale der letzteren gemein haben." (Here the $p$ 's are power series in $(x-\theta)$ such that $p_{0}(\theta), \cdots, p_{n}(\theta)$ are all $\neq 0$; cf. $(B)$, appendix.)

This theorem falls in $\mathfrak{I}_{+1}$, Situation 10 . We have

$$
\begin{array}{r}
\gamma_{0}=-(n+2), \quad \cdots, \quad \gamma_{\nu}=-\left(n-\nu+\mu_{\nu}\right), \quad \cdots, \quad \gamma_{n}=0 \\
\left(\gamma_{n}-\gamma_{0}\right) / n=(n+2) / n>\left(\nu+2-\mu_{\nu}\right) / \nu=\left(\gamma_{\nu}-\gamma_{0}\right) / \nu \\
\quad\{\nu=1,2, \cdots, n-1\} .
\end{array}
$$

Hence, according to the "Criterion for Irreducibility," $C$ is irreducible if $n$ is odd; and according to the "Factorization Theorem," if $n$ is even, the order of a factor of $C$ is of the form $i n / 2$, where $i$ is an integer. This result is equivalent to the statement of the theorem.

(b) P. 73. "Eine Differentialgleichung von der Form

$$
\begin{aligned}
& (x-\theta)^{n+\mu_{0}} p_{0}(x) \frac{d^{n} y}{d x^{n}}+(x-\theta)^{n-1+\mu_{1}} p_{1}(x) \frac{d^{n-1} y}{d x^{n-1}}+\cdots \\
& +(x-\theta)^{1+\mu_{n-1}} p_{n-1}(x) \frac{d y}{d x}+p_{n}(x) y=0,
\end{aligned}
$$

in welcher $\mu_{1}, \mu_{2}, \cdots, \mu_{n-1} \geqq \mu_{0}$ sind, kann nur mit einer gleichartigen Differentialgleichung, deren Ordnung $\geqq n / \mu_{0}$ ist, alle Integrale der letzteren gemein haben." (Here the $p$ 's are as in $(a)$.) 
A special case of the "Factorization Theorem," $\mathfrak{T}_{+1}$, Situation 10.

(c) P. 77. "Die lineare Differentialgleichung

$$
\begin{aligned}
& (x-\theta)^{\rho n+1} p_{0}(x) \frac{d^{n} y}{d x^{n}}+(x-\theta)^{\rho(n-1)+\nu_{1}} p_{1}(x) \frac{d^{n-1} y}{d x^{n-1}} \\
& +(x-\theta)^{\rho(n-2)+\nu_{2}} p_{2}(x) \frac{d^{n-2} y}{d x^{n-2}}+\cdots+(x-\theta)^{\rho+\nu_{n-1}} p_{n-1}(x) \frac{d y}{d x} \\
& +p_{n}(x) y=0,
\end{aligned}
$$

in welcher $\nu_{1}, \nu_{2}, \cdots, \nu_{n-1} \geqq 1$ und $\rho$ eine beliebige positive ganze Zahl bedeutet, ist irreductibel." (Here the $p$ 's are as in (a).)

A consequence of the "Criterion for Irreducibility," $\mathfrak{T}_{+1}$, Situation 10.

(G) Koenigsberger, J o u r na l f ür M a the matik, vol. 121, pp. 320-359. The leading results on factorization contained in this paper-in particular, the (complicated but general) result p. 342-fall under the "Factorization Theorem," $\mathfrak{I}_{-\infty}$, either for Situation 1 or for Situation $5, k=1$.

(H) Perron's Criterion, J o u nal für Mathematik, vol. 132, p. 304.

"Wenn sich unter den respektiven Gradzahlen $\gamma_{1}, \gamma_{2}, \cdots, \gamma_{n}$ der Funktionen $c_{1}(x), c_{2}(x), \cdots, c_{n}(x)$ eine findet $\left(\gamma_{i}\right)$, welche den Ungleichungen

$$
\begin{aligned}
& \text { (a) } \gamma_{i}>\gamma_{\nu} \\
& \text { (.) } \frac{\gamma_{i}}{i}>\frac{\gamma_{\nu}}{\nu}
\end{aligned}
$$

genügt, wo $\nu$ jede von $i$ verschiedene $\operatorname{der}$ Zahlen $1,2, \cdots, n$ bedeutet; und wenn ausserdem $\gamma_{i}$ relativ prim ist zu $i$, so ist

$$
C=f(x, y)=y^{n}+c_{1}(x) y^{n-1}+\cdots+c_{n}(x)
$$

irreduzibel. Sind aber die Ungleichungen $(\alpha)$ nur insoweit erfüllt, dass für gewisse Werte von $\nu$ das Gleichheiszeichen an Stelle des Grösserzeichens tritt, so ist für die Irreduzibilität ausserdem noch erforderlich, dass keine Konstante $c$ existiert, für die $f(x, c)$ als Funktion von $x$ identisch verschwindet." (The $c$ 's are here polynomials in $x$ with arbitrary complex coefficients.)

This theorem is a consequence of the "Theorem on the Product," $\mathfrak{I}_{-\infty}$, Situation $3, k=1, x_{1}=x$. For, from $(\beta)$ it follows, since $\gamma_{0}=0$, that the first element $\left(m_{1}, t_{1}\right)$ of the characteristic set of $C$ is $\left(\gamma_{i} / i, i\right)$. If $i=n$, it follows from the "Criterion for Irreducibility," since $\gamma_{i}$ and $i$ are relatively prime, that $C$ is irreducible. If $i<n$, the characteristic set of $C$ has other elements $\left(m_{\zeta}, t_{\zeta}\right)$, where $\zeta>1$, but on account of $(\alpha), m_{\zeta}$ must be $<0$. Now, according to the "Theorem on the Product" [cf. proof of the "Factorization Theorem "], it follows in virtue of the relative primeness of $\gamma_{i}$ 
and $i$, that only one of the factors, say $A$, of $C=A \cdot B(r \geqq 1$ and $s \geqq 1)$ can have $m_{i}=\gamma_{i} / i$ as an $m$ of its characteristic set; and every $m$ of the characteristic set of $B$ is thus $<0$. According to a theorem of Gauss, we may assume, without loss of generality, that the coefficients of the various powers of $y$ in $B$ are polynomials in $x$ and hence of rank $\geqq 0$. Since $\beta_{0}$ ( $=$ rank of the coefficient of the highest power of $y$ in $B$ ) is then obviously 0 , at least one $m$ of the characteristic set of $B$ is $\geqq 0$. This contradiction shows that $C$ is irreducible. If, on the other hand, the inequality $(\alpha)$ is changed to $\gamma_{i} \geqq \gamma_{\nu}$, we have no longer a contradiction, but can merely conclude that every $m$ of the characteristic set of $B$ is 0 . Hence the rank of every coefficient of the powers of $y$ in $B$ is 0 ; that is, these coefficients are constants. Tharefore, $B$ depends on $y$ alone, and there exists a value $c$ for $y$, namely a root for $y$ of $B=0$, such that $C=f(x, c)=A \cdot B \equiv 0$.

(I) Dumas, Journal de Mathématiques, ser. 6, vol. 2, pp. 191-258. The theorem p. 217 is equivalent to the "Theorem on the Product "; the theorem p. 237, to the "Factorization Theorem "; and the theorem p. 242, to the "Theorem on Common Divisors," all for $\mathfrak{I}_{-\infty}$, Situation 2 .

( $J$ ) Bauer, J o u rnal für Mathe matik (see previous citations). The principal results come under the results of Dumas.

UNIVERSITY OF NEBRASKA,

Dec. 30, 1915. 